\title{
THE STRESS INTENSITY FACTORS OF REGULARLY PERTURBED-INTERFACE CRACKS OF ANISOTROPIC BIMATERIALS
}

\author{
CHAO-HSUN CHEN and JINHON HSU \\ Institute of Applied Mechanics, National Taiwan University, Taiwan, Republic of China
}

(Received 12 September 1995 ; in revised form 10 April 1996)

\begin{abstract}
Based on Lekhnitskii-Eshelby-Stroh (LES) representation and perturbation analysis, analytic solutions are given for displacement and stress fields of two anisotropic half-planes, forming a composite bimaterial, with a perturbed-interface crack. Among various mathematical models representing real cracks, the "thin cut" model is of special interest, since it requires the simplest mathematical methods in its study. However, the model does not refiect some of the properties of actual cracks, in particular the crack should be uneven. When the lateral stresses, parallel to the interface, dominate in the fracture mechanism, the thin-cut model cannot reveal any stress intensifying phenomenon, while many failures, occurring in the interfaces of thin-film and substrate or fiber and matrix, are always induced by crucial lateral stresses. For these reasons, the unevenness effect of crack faces must be taken into account to determine the practical stress intensity factors for predicting the interface fracture behavior. A modified crack with smoothly perturbed surfaces ensures good agreement with reality, while retaining the simplicity of the mathematical model. Mathematically, we consider the elastic problem of a perturbed-interface crack lying along the interface of two bonded dissimilar anisotropic half-planes and the uniform far-field stresses are specified. When the lateral stresses are much larger than others, the solutions are determined to the first-order of unevenness to understand how the lateral stresses affect the stress intensity factors as the crack face is uneven. (C) 1997 Elsevier Science Ltd. All rights reserved.
\end{abstract}

\section{INTRODUCTION}

The fact motivating the present work is that the interface structures of fiber and matrix or thin film and substrate will always fail or debond under certain crucial lateral stresses. This fracture behavior is very difficult to be analyzed, if the interface crack is traditionally assumed to be a flat thin-cut. Mathematically, the stress intensity factors are never affected by the lateral stresses, provided that the interface crack is a thin-cut. In this paper, this difficulty can be solved by assuming that the interface cracks are slightly perturbed from a straight line and the stress intensity factors are also presented to show the effect induced by the lateral stress. In a recent paper, Chen and Hsu (1995) presented analytic solutions for perturbed-interface cracks in isotropic half-spaces consisting of dissimilar materials. In the present paper, we will extend the study to the anisotropic bimaterials.

The configuration of the interesting problem, with a non-dimensionalized Cartesian coordinate system $\left(x_{1}, x_{2}\right)$, is depicted in Fig. 1. The interface is outlined by two straight bonded lines and a perturbed-interface crack, which are defined mathematically by

$$
\begin{gathered}
C_{\mathrm{b}}: x_{2}=0 ; x_{1} \in(-\infty,-1] \cup[1, \infty) \quad \text { bonded lines, } \\
C_{\mathrm{u}}: x_{2}=\varepsilon Y_{+}\left(x_{1}\right) ; x_{1} \in(-1,1) \quad \text { upper surface of crack, } \\
C_{1}: x_{2}=\varepsilon Y_{-}\left(x_{1}\right) ; x_{1} \in(-1,1) \quad \text { lower surface of crack, }
\end{gathered}
$$

where

$$
\begin{gathered}
Y_{+}\left(x_{1}\right)-Y_{-}\left(x_{1}\right) \geqslant 0, \\
Y_{ \pm}( \pm 1)=0
\end{gathered}
$$




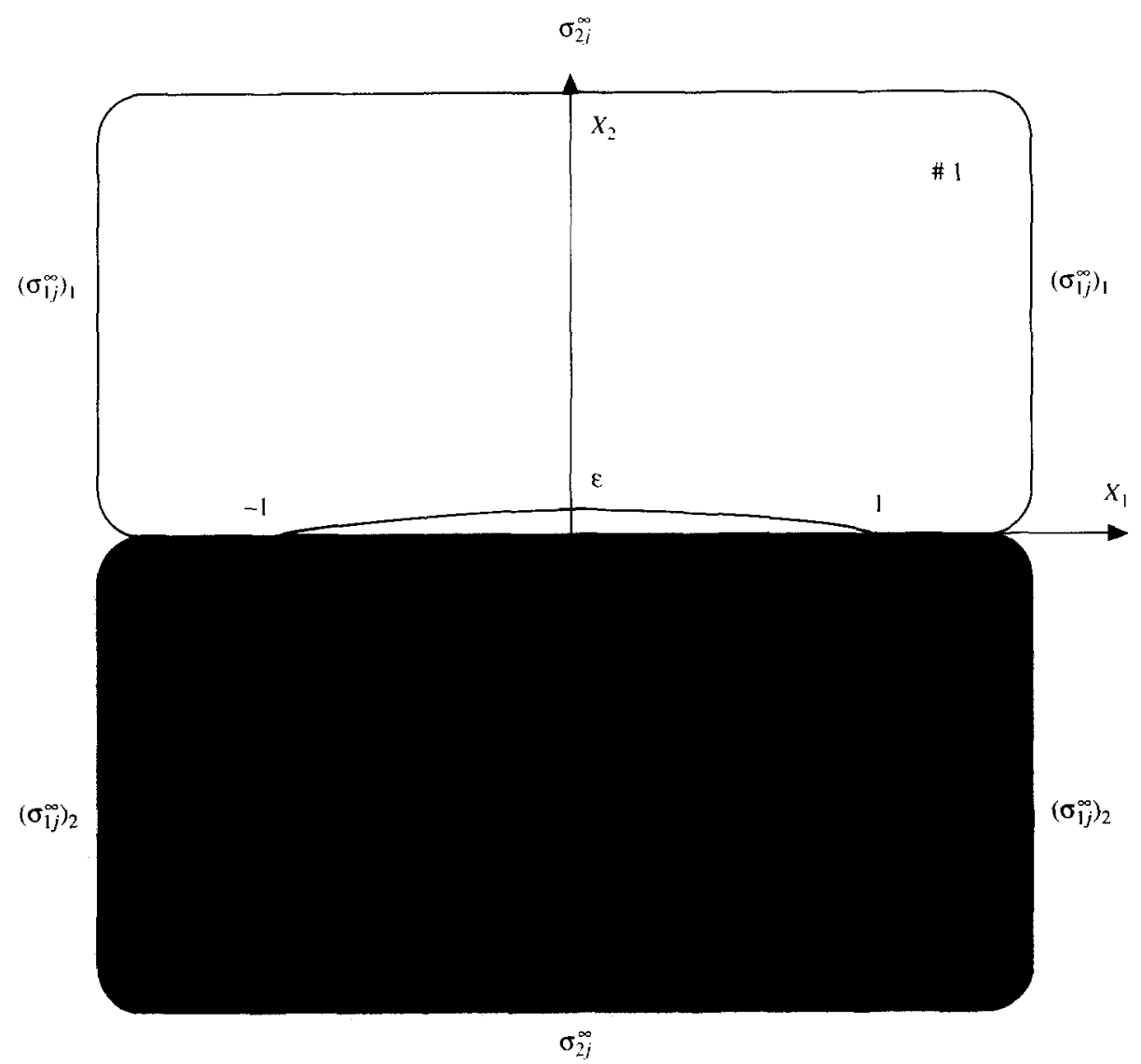

Fig. 1. Schematic of regularly perturbed-interface crack between anisotropic bimaterials.
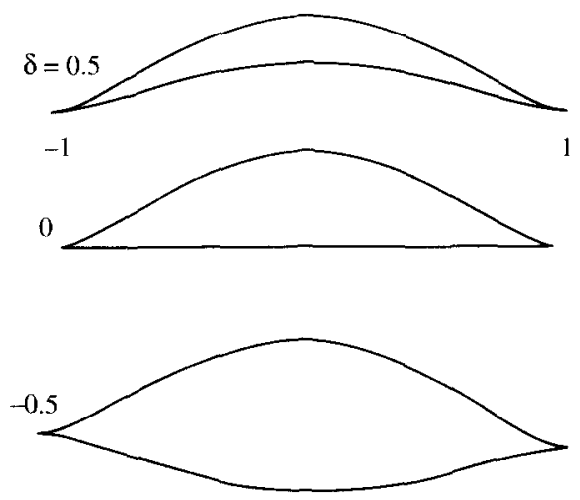

Fig. 2. Schematics of holes with cusps.

and $\varepsilon$ is a small parameter, which is so designed in (2) and (3) that the unevenness of the crack faces is slight. As $\varepsilon$ tends to zero, the perturbed-interface crack degenerates into a straight crack of length 2 . The functions $Y_{ \pm}$, together with their derivatives, are designated to be continuous in order to avoid any additional singularity except at $x_{1}= \pm 1$.

A perturbed-interface crack is called "regular" if

$$
Y_{ \pm}^{\prime}( \pm 1)=0,
$$

which ensures the existence of cusps at $x= \pm 1$. For such a case, the perturbation procedure used is a regular one in that all asymptotic expressions are uniformly valid throughout the region of interest. 
LES representation, presented by Suo (1990) and perturbation analysis are the basic concepts which were used to formulate and study the problem considered in this paper. Homogeneous and general Hilbert's problems are obtained as in Clements (1971) for solving the perturbed-interface cracks, but the functions $Y_{ \pm}$appearing in the Hilbert's problems must be known a priori, otherwise only complicated integral forms could be found. Hence, a special case simulating practical failure is chosen to demonstrate the solving process and give some referable results. The corresponding stress intensity factor will be determined to the first-order as the crucial lateral stresses dominate the failure.

\section{LEKHNITSKII-ESHELBY-STROH (LES) REPRESENTATION}

The equivalence of those somewhat different formulations used by Lekhnitskii (1963), Eshelby et al. (1953) and Stroh (1958) in solving anisotropic elasticity problems was proved by Suo (1990). Referring to this LES representation, the interesting problem considered in the present paper could be formulated with the following equations for displacements $u_{i}$, stresses $\sigma_{i j}$ and resultant forces on an arc $T_{i}$.

$$
\begin{gathered}
\mathbf{u}_{\alpha}(z)=\left\{u_{i}(z)\right\}_{\alpha}=\mathbf{A}_{\alpha} \mathbf{f}_{\alpha}(z)+\overline{\mathbf{A}_{\alpha}} \overline{\mathbf{f}_{\alpha}(z)}, \\
\mathbf{T}_{\alpha}(z)=\left\{T_{i}(z)\right\}_{\alpha}=-\mathbf{L}_{\alpha} \mathbf{f}_{\alpha}(z)-\overline{\mathbf{L}_{\alpha}} \overline{\mathbf{f}_{\alpha}(z)}, \\
\mathbf{t}_{\alpha}(z)=\left\{\sigma_{2 j}(z)\right\}_{\alpha}=\mathbf{L}_{\alpha} \mathbf{f}_{\alpha}^{\prime}(z)+\overline{\mathbf{L}_{\alpha}} \mathbf{f}_{\alpha}^{\prime}(z), \\
\mathbf{s}_{\alpha}(z)=\left\{\sigma_{1 j}(z)\right\}_{\alpha}=-\mathbf{L}_{\alpha}\left\langle\left\langle\mu_{\alpha}\right\rangle\right\rangle \mathbf{f}_{\alpha}^{\prime}(z)-\overline{\mathbf{L}_{\alpha}}\left\langle\left\langle\overline{\mu_{\alpha}}\right\rangle\right\rangle \overline{\mathbf{f}_{\alpha}^{\prime}(z)} .
\end{gathered}
$$

Here and generally in this paper, Greek suffixes $\alpha$ could be 1 or 2 as a material index indicating that the field quantities belong to upper $(\alpha=1)$ or lower $(\alpha=2)$ half planes and Latin suffix $i$ and $j$ could be 1,2 or 3 as component indices. The vector function $\mathbf{f}_{\alpha}(z)=\left[f_{1}\left(z_{1}\right), f_{2}\left(z_{2}\right), f_{3}\left(z_{3}\right)\right]_{\alpha}^{\mathrm{T}}$; superscript $\mathrm{T}$ stands for transpose, is holomorphic in the arguments $z_{j}=x+\mu_{j} y$ and $u_{j}$ are three distinct complex numbers with positive imaginary parts, which can be solved as roots of the sixth-order polynomial shown below.

$$
l_{2}(\mu) l_{4}(\mu)-\left[l_{3}(\mu)\right]^{2}=0
$$

where

$$
\begin{aligned}
& l_{2}(\mu)=s_{55} \mu^{2}-2 s_{45} \mu+s_{44}, \\
& l_{4}(\mu)=s_{11} \mu^{4}-2 s_{16} \mu^{3}+\left(2 s_{12}+s_{66}\right) \mu^{2}-2 s_{26} \mu+s_{22}, \\
& l_{3}(\mu)=s_{15} \mu^{3}-\left(s_{14}+s_{56}\right) \mu^{2}+\left(s_{25}+s_{46}\right) \mu-s_{24}
\end{aligned}
$$

and $s_{p q} ; p, q=1-6$ are conventional compliancies to relate the stress vector $\left\{\sigma_{q}\right\}=\left[\sigma_{11}, \sigma_{22}, \sigma_{33}, \sigma_{23}, \sigma_{31}, \sigma_{12}\right]^{\mathrm{T}}$ and the strain vector $\left\{\varepsilon_{p}\right\}=\left[\varepsilon_{11}, \varepsilon_{22}, \varepsilon_{33}, 2 \varepsilon_{23}, 2 \varepsilon_{31}, 2 \varepsilon_{12}\right]^{\mathrm{T}}$ as a Hooke's law. (11) had been proved to have no real root, and the roots are also assumed to be distinct in the present paper as they were in some other literatures. The six roots form three complex conjugate pairs, from which three $\mu_{j}$ with positive imaginary parts can be selected. Also $\mu_{\alpha}=\left[\mu_{1}, \mu_{2}, \mu_{3}\right]_{\alpha}^{\mathrm{T}},\left\langle\left\langle\mu_{\alpha}\right\rangle\right\rangle=\operatorname{diag}\left[\mu_{1}, \mu_{2}, \mu_{3}\right]_{\alpha}$; diag[ $]$ denotes a diagonal matrix. The elements of the matrices $A$ and $L$ are given by

$$
\mathbf{L}=\left[\begin{array}{ccc}
-\mu_{1} & -\mu_{2} & -\mu_{3} \eta_{3} \\
1 & 1 & \eta_{3} \\
-\eta_{1} & -\eta_{2} & -1
\end{array}\right]
$$

and 


$$
\begin{aligned}
& \mathbf{A}_{1 \beta}=s_{11} \mu_{\beta}^{2}+s_{12}-s_{16} \mu_{\beta}+\eta_{\beta}\left(s_{15} \mu_{\beta}-s_{14}\right), \\
& \mathbf{A}_{2 \beta}=s_{21} \mu_{\beta}+s_{12} / \mu_{\beta}-s_{26}+\eta_{\beta}\left(s_{25}-s_{24} / \mu_{\beta}\right), \\
& \mathbf{A}_{3 \beta}=s_{41} \mu_{\beta}+s_{42} / \mu_{\beta}-s_{46}+\eta_{\beta}\left(s_{45}-s_{44} / \mu_{\beta}\right),
\end{aligned}
$$

for $\beta=1,2$ and

$$
\begin{aligned}
\mathbf{A}_{13} & =\eta_{3}\left(s_{11} \mu_{3}^{2}+s_{12}-s_{16} \mu_{3}\right)+s_{15} \mu_{3}-s_{14}, \\
\mathbf{A}_{23} & =\eta_{3}\left(s_{21} \mu_{3}+s_{22} / \mu_{3}-s_{26}\right)+s_{25}-s_{24} / \mu_{3}, \\
\mathbf{A}_{33} & =\eta_{3}\left(s_{41} \mu_{3}+s_{42} / \mu_{3}-s_{46}\right)+s_{45}-s_{44} / \mu_{3},
\end{aligned}
$$

where

$$
\eta_{\beta}=-l_{3}\left(\mu_{\beta}\right) / l_{2}\left(\mu_{\beta}\right) \quad(\beta=1,2) \quad \eta_{3}=-l_{3}\left(\mu_{3}\right) / l_{4}\left(\mu_{3}\right) .
$$

The matrices $\mathbf{A}$ and $\mathbf{L}$ shown above have been proved to be non-singular under the assumption that the roots of the characteristic equation are distinct and a positive-definite Hermitian matrix $\mathbf{B}$ derived from the inner product of $\mathbf{A}$ and $\mathbf{L}$ has been defined as

$$
\mathbf{B}=\mathbf{i A L ^ { - 1 }}
$$

The matrix B has appeared in many solutions and it is named the surface admittance tensor, the inverse of surface impedance tensor and was investigated in sufficient detail by Barnett and Lothe (1985) to facilitate the analysis of the Rayleigh and Stoneley wave problem. For convenience, a positive-definite Hermitian matrix $\mathbf{H}$ involving bimaterial elastic constants is defined as

$$
\mathbf{H}=\mathbf{B}_{1}+\overline{\mathbf{B}}_{2} .
$$

Throughout the paper, the matrices $\mathbf{B}$ and $\mathbf{H}$ will appear in some of the main solutions.

With the expressions of field quantities in (7)-(10), the perturbed-interface crack problem can be formulated to provide a solution. The structure of anisotropic bimaterial is here subjected to uniform far-field stresses. The interface between the two media involves two parts, one is the bonded part including $x_{1} \leqslant-1$ and $x_{1} \geqslant 1$ to be named $C_{b}$ and the other is the perturbed-interface crack in $-1<x_{1}<1 \times x_{1}$ between -1 and 1 which will be replaced by $x_{1}^{*}$ in the following sections of this paper to distinguish the perturbed part from the bonded part. It is assumed that along $C_{b}$ the interface is perfectly bonded so that we obtain two continuity equations as shown in (16) and (17).

$$
\begin{aligned}
& \mathbf{T}_{1}(z)=\mathbf{T}_{2}(z) \text {; i.e. } \mathbf{L}_{1} \mathbf{f}_{1}(z)+\overline{\mathbf{L}_{1}} \overline{\mathbf{f}_{1}(z)}=\mathbf{L}_{2} \mathbf{f}_{2}(z)+\overline{\mathbf{L}_{2}} \overline{\mathbf{f}_{2}(z)}, \\
& \mathbf{u}_{1}(z)=\mathbf{u}_{2}(z) \text {; i.e. } \mathbf{A}_{1} \mathbf{f}_{1}(z)+\overline{\mathbf{A}_{1}} \overline{\mathbf{f}_{1}(z)}=\mathbf{A}_{2} \mathbf{f}_{2}(z)+\overline{\mathbf{A}_{2}} \overline{\mathbf{f}_{2}(z)}
\end{aligned}
$$

The continuity equation (17) can be rearranged with the aid of (16) as

$$
\mathbf{L}_{1} \mathbf{f}_{1}(z)=\mathbf{H}^{-1}\left(\mathbf{B}_{2}+\overline{\mathbf{B}_{2}}\right) \mathbf{L}_{2} \mathbf{f}_{2}(z)+\mathbf{H}^{-1}\left(\overline{\mathbf{B}_{1}}-\overline{\mathbf{B}_{2}}\right) \overline{\mathbf{L}_{1}} \overline{\mathbf{f}_{1}(z)} .
$$

Besides, the traction-free condition is assumed along the crack faces. From (8) we then have two condition equations :

$$
\mathbf{L}_{1} \mathbf{f}_{1}(z)+\overline{\mathbf{L}_{1}} \overline{\mathbf{f}_{1}(z)}=0
$$

$$
\mathbf{T}_{1}(z)=\mathbf{T}_{2}(z)=0
$$

$$
\mathbf{L}_{2} \mathbf{f}_{2}(z)+\overline{\mathbf{L}_{2}} \overline{\mathbf{f}_{2}(z)}=0,
$$

where $z$ is along the crack faces. 


\section{PERTURBATION ANALYSIS}

Based on the perturbation theory, the complex function vectors $f_{\alpha}(z)$ could be rewritten as $f_{a}(z ; \varepsilon)$ after involving the perturbation variable $\varepsilon$ and they can be expressed in powers of $\varepsilon$ as

$$
\mathbf{f}_{\alpha}(z)=\mathbf{f}_{\alpha}(z ; \varepsilon) \sim \sum_{n=0}^{\infty} \frac{\varepsilon^{n}}{n !} \mathbf{f}_{\alpha n}(z)=\mathbf{f}_{\alpha 0}(z)+\varepsilon \mathbf{f}_{\alpha 1}(z)+, \cdots
$$

With (21), the continuity conditions (16) and (18) along the bonded portions of the interface can be expanded as

$$
\begin{aligned}
\mathbf{L}_{1}\left(\mathbf{f}_{10}^{+}\left(x_{1}\right)+\varepsilon \mathbf{f}_{11}^{+}\left(x_{1}\right)+\right. & \left., \cdots,)+\overline{\mathbf{L}_{1}} \overline{\left(\mathbf{f}_{10}^{-}\right.}\left(x_{1}\right)+\varepsilon \overline{\mathbf{f}_{11}^{-}}\left(x_{1}\right)+, \cdots,\right) \\
& =\mathbf{L}_{2}\left(\mathbf{f}_{20}^{+}\left(x_{1}\right)+\varepsilon \mathbf{f}_{21}^{+}\left(x_{1}\right)+, \cdots,\right)+\overline{\mathbf{L}_{2}}\left(\overline{\mathbf{f}_{20}^{-}}\left(x_{1}\right)+\varepsilon \overline{\mathbf{f}_{21}^{-}}\left(x_{1}\right)+, \cdots,\right)
\end{aligned}
$$

and

$$
\begin{aligned}
\mathbf{L}_{1}\left(\mathbf{f}_{10}^{+}\left(x_{1}\right)+\varepsilon \mathbf{f}_{11}^{+}\left(x_{1}\right)+, \cdots,\right)=\mathbf{H}^{-1}\left(\mathbf{B}_{2}\right. & \left.+\overline{\mathbf{B}_{2}}\right) \mathbf{L}_{2}\left(\mathbf{f}_{20}^{+}\left(x_{1}\right)+\varepsilon \mathbf{f}_{21}^{+}\left(x_{1}\right)+, \cdots,\right) \\
& +\mathbf{H}^{-1}\left(\overline{\mathbf{B}_{1}}-\overline{\left.\mathbf{B}_{2}\right)} \overline{\mathbf{L}_{1}}\left(\overline{\mathbf{f}_{10}^{-}}\left(x_{1}\right)+\varepsilon \overline{\mathbf{f}_{11}^{-}}\left(x_{1}\right)+, \cdots,\right),\right.
\end{aligned}
$$

where

$$
\begin{aligned}
& \mathbf{f}_{1 n}^{+}\left(x_{1}\right)=\lim _{x_{2} \rightarrow 0^{ \pm}} \mathbf{f}_{1 n}(z), \overline{\mathbf{f}_{1 n}^{\mp}}\left(x_{1}\right)=\lim _{x_{2} \rightarrow 0^{ \pm}} \overline{\mathbf{f}_{1 n}(z)}, \\
& \mathbf{f}_{2 n}^{+}\left(x_{1}\right)=\lim _{x_{2} \rightarrow 0} \mathbf{f}_{2 n}(z), \overline{\mathbf{f}_{2 \hbar}}\left(x_{1}\right)=\lim _{x_{2} \rightarrow 0} \overline{\mathbf{f}_{2 n}(z)} .
\end{aligned}
$$

The upper and lower crack faces are simulated by $Y_{+}\left(x_{1}^{*}\right)$ and $Y_{-}\left(x_{1}^{*}\right)$, respectively, such that along them the arguments of the complex functions will be

$$
\text { on } Y_{+} \quad z_{j}=z_{j}^{*}=x_{1}^{*}+\mu_{j} x_{2}=x_{1}^{*}+\varepsilon \mu_{j} Y_{+}\left(x_{1}^{*}\right) ; \mu_{j} \in \mu_{1}
$$

and

$$
\text { on } Y_{-} \quad z_{j}=z_{j}^{*}=x_{1}^{*}+\mu_{j} x_{2}=x_{1}^{*}+\varepsilon \mu_{j} Y_{-}\left(x_{1}^{*}\right) ; \mu_{j} \in \mu_{2} .
$$

Thus, the functions $\mathbf{f}_{1 n}\left(z^{*}\right)$ and $\mathbf{f}_{2 n}\left(z^{*}\right)$ can be expanded as follows:

$$
\mathbf{f}_{1 n}\left(z^{*}\right)=\mathbf{f}_{1 n}^{+}\left(x_{1}^{*}\right)+\varepsilon Y_{+}\left(x_{1}^{*}\right)\left\langle\left\langle\mu_{1}\right\rangle\right\rangle \mathbf{f}_{1 n}^{\prime+}\left(x_{1}^{*}\right)+O\left(\varepsilon^{2}\right)
$$

and

$$
\mathbf{f}_{2 n}\left(z^{*}\right)=\mathbf{f}_{2 n}^{+}\left(x_{1}^{*}\right)+\varepsilon Y_{-}\left(x_{1}^{*}\right)\left\langle\left\langle\mu_{2}\right\rangle\right\rangle \mathbf{f}_{2 n}^{+}\left(x_{1}^{*}\right)+O\left(\varepsilon^{2}\right) .
$$

The condition equations (19) and (20) are then expanded with the aid of (27) and (28) along the crack faces.

$$
\begin{aligned}
\mathbf{L}_{1}\left\{\mathbf{f}_{10}^{+}\left(x_{1}^{*}\right)+\varepsilon\left[Y_{+}\left(x_{1}^{*}\right)\right.\right. & \left.\left.\left\langle\left\langle\mu_{1}\right\rangle\right\rangle \mathbf{f}_{10}^{+}\left(x_{1}^{*}\right)+\mathbf{f}_{11}^{+}\left(x_{1}^{*}\right)\right]+O\left(\varepsilon^{2}\right)\right\} \\
& +\overline{\mathbf{L}_{1}}\left\{\overline{\mathbf{f}_{10}^{-}}\left(x_{1}^{*}\right)+\varepsilon\left[Y_{+}\left(x_{1}^{*}\right)\left\langle\left\langle\overline{\mu_{1}}\right\rangle \overline{\mathbf{f}_{10}^{-}}\left(x_{1}^{*}\right)+\overline{\mathbf{f}_{11}^{-}}\left(x_{1}^{*}\right)\right]+O\left(\varepsilon^{2}\right)\right\}=0\right.
\end{aligned}
$$

and 
$\mathbf{L}_{2}\left\{\mathbf{f}_{20}^{+}\left(x_{1}^{*}\right)+\varepsilon\left[Y_{-}\left(x_{1}^{*}\right)\left\langle\left\langle\mu_{2}\right\rangle\right\rangle \mathbf{f}_{20}^{+}\left(x_{1}^{*}\right)+\mathbf{f}_{21}^{+}\left(x_{1}^{*}\right)\right]+O\left(\varepsilon^{2}\right)\right\}$

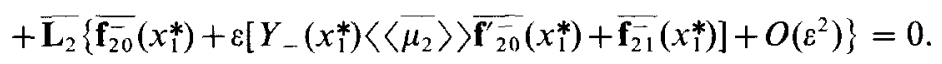

From (22), (23), (29) and (30), we have two groups of equations corresponding to the orders of $\varepsilon^{0}$ and $\varepsilon^{1}$ as follows :

$$
\begin{gathered}
\varepsilon^{v} \mathbf{L}_{1} \mathbf{f}_{10}^{+}\left(x_{1}\right)+\overline{\mathbf{L}_{1}} \overline{\mathbf{f}_{10}^{-}}\left(x_{1}\right)=\mathbf{L}_{2} \mathbf{f}_{20}^{+}\left(x_{1}\right)+\overline{\mathbf{L}_{2}} \overline{\mathbf{f}_{20}}\left(x_{1}\right), \\
\mathbf{L}_{1} \mathbf{f}_{10}^{+}\left(x_{1}\right)=\mathbf{H}^{-1}\left(\mathbf{B}_{2}+\mathbf{B}_{2}\right) \mathbf{L}_{2} \mathbf{f}_{20}^{+}\left(x_{1}\right)+\mathbf{H}^{-1}\left(\overline{\mathbf{B}_{1}^{-}}-\overline{\mathbf{B}_{2}^{-}}\right) \mathbf{L}_{1} \mathbf{f}_{10}^{-}\left(x_{1}\right), \\
\mathbf{L}_{1} \mathbf{f}_{10}^{+}\left(x_{1}^{*}\right)+\overline{\mathbf{L}_{1}} \overline{\mathbf{f}_{10}^{-}}\left(x_{1}^{*}\right)=0
\end{gathered}
$$

and

$$
\begin{aligned}
& \mathbf{L}_{2} \mathbf{f}_{20}^{+}\left(x_{1}^{*}\right)+\overline{\mathbf{L}_{2}} \overline{\mathbf{f}_{20}^{-}}\left(x_{1}^{*}\right)=0, \\
& \varepsilon^{1} \mathbf{L}_{1} \mathbf{f}_{11}^{+}\left(x_{1}\right)+\overline{\mathbf{L}_{1}} \overline{\mathbf{f}_{11}^{-}}\left(x_{1}\right)=\mathbf{L}_{2} \mathbf{f}_{21}^{+}\left(x_{1}\right)+\overline{\mathbf{L}_{2}} \overline{\mathbf{f}_{21}^{-}}\left(x_{1}\right), \\
& \mathbf{L}_{1} \mathbf{f}_{11}^{+}\left(x_{1}\right)=\mathbf{H}^{-1}\left(\mathbf{B}_{2}+\overline{\mathbf{B}_{2}}\right) \mathbf{L}_{2} \mathbf{f}_{21}^{+}\left(x_{1}\right)+\mathbf{H}^{-1}\left(\overline{\mathbf{B}_{1}}-\overline{\mathbf{B}_{2}}\right) \overline{\mathbf{L}_{1}} \overline{\mathbf{f}_{11}^{-}}\left(x_{1}\right) \\
& \mathbf{L}_{1} \mathbf{f}_{11}^{+}\left(x_{1}^{*}\right)+\overline{\mathbf{L}_{1}} \overline{\mathbf{f}_{11}^{-}}\left(x_{1}^{*}\right)+Y_{+}\left(x_{1}^{*}\right)\left[\mathbf{L}_{1}\left\langle\left\langle\mu_{1}\right\rangle\right\rangle \mathbf{f}_{10}^{\prime+}\left(x_{1}^{*}\right)+\overline{\mathbf{L}_{1}}\left\langle\left\langle\overline{\mu_{1}}\right\rangle\right\rangle \overline{\mathbf{f}_{10}^{\prime}}\left(x_{1}^{*}\right)\right]=0
\end{aligned}
$$

and

$$
\mathbf{L}_{2} \mathbf{f}_{21}^{+}\left(x_{1}^{*}\right)+\overline{\mathbf{L}_{2}} \overline{\mathbf{f}_{21}^{-}}\left(x_{1}^{*}\right)+Y_{-}\left(x_{1}^{*}\right)\left[\mathbf{L}_{2}\left\langle\left\langle\mu_{2}\right\rangle\right\rangle \mathbf{f}_{20}^{+}\left(x_{1}^{*}\right)+\overline{\mathbf{L}_{2}}\left\langle\left\langle\overline{\left\langle\mu_{2}\right.}\right\rangle\right\rangle \overline{\mathbf{f}_{20}^{\prime}}\left(x_{1}^{*}\right)\right]=0,
$$

where eqns (31), (32), (35) and (36) come from the continuity conditions along the bonded interface and eqns (33), (34), (37) and (38) from the traction-free conditions on the crack faces.

\section{BASIC SOLUTION TO THE ORDER OF $\varepsilon^{0}$}

Interface cracks without perturbed faces in anisotropic materials have been discussed by many authors [e.g. Gotoh (1967), Clements (1971), Willis (1971), Ting (1986, 1990), Bassani and Qu (1989), Tewary et al. (1989), Suo (1990), Wu (1990), Ni and Nemat Nasser (1991) and Qu and Li (1991)]. The solution obtained in this section has a similar result to those presented by those authors, but the applied stresses are remotely located. The solution is referred to as the zeroth order effect in perturbation analysis.

Equations (31), (32), (35) and (36) can be incorporated as follows:

$$
\mathbf{L}_{1} \mathbf{f}_{1 k}^{+}\left(x_{1}\right)-\overline{\mathbf{L}_{2}} \overline{\mathbf{f}_{2 k}}\left(x_{1}\right)=\mathbf{L}_{2} \mathbf{f}_{2 k}^{+}\left(x_{1}\right)-\overline{\mathbf{L}_{1}} \overline{\mathbf{f}_{1 k}}\left(x_{1}\right)
$$

and

$$
\mathbf{L}_{1} \mathbf{f}_{1 k}^{+}\left(x_{1}\right)=\mathbf{H}^{-1}\left(\mathbf{B}_{2}+\overline{\mathbf{B}_{2}}\right) \mathbf{L}_{2} \mathbf{f}_{2 k}^{+}\left(x_{1}\right)+\mathbf{H}^{-1}\left(\overline{\mathbf{B}_{1}}-\overline{\mathbf{B}_{2}}\right) \overline{\mathbf{L}_{1}} \overline{\mathbf{f}_{1 k}^{-}}\left(x_{1}\right),
$$

where $k=0,1$.

Keeping (39) and (40) in mind, two new complex vector functions $\Theta_{k}(z)$ and $\Phi_{k}(z)$ are designated to be 


$$
\begin{aligned}
\Theta_{k}(z) & =\mathbf{L}_{1} \mathbf{f}_{1 k}(z)-\overline{\mathbf{L}_{2}} \overline{\mathbf{f}_{2 k}}(z) \\
& =\mathbf{L}_{2} \mathbf{f}_{2 k}(z)-\overline{\mathbf{L}_{1}} \overline{\mathbf{f}_{1 k}}(z)
\end{aligned}
$$

and

$$
\begin{aligned}
\Phi_{k}(z) & =\mathbf{L}_{1} \mathbf{f}_{1 k}(z) \\
& =\mathbf{H}^{-1}\left(\mathbf{B}_{2}+\overline{\mathbf{B}_{2}}\right) \mathbf{L}_{2} \mathbf{f}_{2 k}(z)+\mathbf{H}^{-1}\left(\overline{\mathbf{B}_{1}}-\overline{\mathbf{B}_{2}}\right) \overline{\mathbf{L}_{1}} \overline{\mathbf{f}_{1 k}}(z) .
\end{aligned}
$$

With the aids of (39) and (40), $\Theta_{k}(z)$ and $\Phi_{k}(z)$ can be proved to satisfy the equalities $\Theta_{k}^{+}\left(x_{1}\right)=\Theta_{k}^{-}\left(x_{1}\right)$ and $\Phi_{k}^{+}\left(x_{1}\right)=\Phi_{k}^{-}\left(x_{1}\right)$. Then, we can conclude that $\Theta_{k}(z)$ and $\Phi_{k}(z)$ are holomorphic vector functions in the whole complex plane cut along -1 to 1 on real axis and the functions $\mathbf{f}_{1 k}(z)$ and $\mathbf{f}_{2 k}(z)$ can be expressed in terms of $\Theta_{k}(z)$ and $\Phi_{k}(z)$.

$$
\begin{gathered}
\mathbf{L}_{1} \mathbf{f}_{1 k}(z)=\Phi_{k}(z), \\
\overline{\mathbf{L}_{1}} \overline{\mathbf{f}_{1 k}}(z)=\overline{\mathbf{H}}^{-1} \mathbf{H} \Phi_{k}(z)-\overline{\mathbf{H}}^{-1}\left(\overline{\mathbf{B}_{2}}+\overline{\mathbf{B}_{2}}\right) \Theta_{k}(z), \\
\mathbf{L}_{2} \mathbf{f}_{2 k}(z)=\overline{\mathbf{H}}^{-1} \mathbf{H} \Phi_{k}(z)+\overline{\mathbf{H}}^{-1}\left(\overline{\mathbf{B}_{1}}-\overline{\mathbf{B}_{2}}\right) \Theta_{k}(z)
\end{gathered}
$$

and

$$
\overline{\mathbf{L}_{2}} \overline{\mathbf{f}_{2 k}}(z)=\Phi_{k}(z)-\Theta_{k}(z) .
$$

Manipulations between (33) and (34) will show us two relation equations of $\Theta_{0}^{+}\left(x_{1}^{*}\right)$ and $\Theta_{0}^{-}\left(x_{1}^{*}\right)$ and of $\Phi_{0}^{+}\left(x_{1}^{*}\right)$ and $\Phi_{0}^{-}\left(x_{1}^{*}\right)$. Equation (33) subtracted from (34) yields

$$
\left[\mathbf{L}_{1} \mathbf{f}_{10}^{+}\left(x_{1}^{*}\right)-\overline{\mathbf{L}_{2}} \overline{\mathbf{f}_{20}^{-}}\left(x_{1}^{*}\right)\right]-\left[\mathbf{L}_{2} \mathbf{f}_{20}^{+}\left(x_{1}^{*}\right)-\overline{\mathbf{L}_{1}} \overline{\mathbf{f}_{10}^{-}}\left(x_{1}^{*}\right)\right]=0,
$$

and adding the two eqns (33) and (34) yields

$$
\mathbf{L}_{1} \mathbf{f}_{10}^{+}\left(x_{1}^{*}\right)+\overline{\mathbf{L}_{1}} \overline{\mathbf{f}_{10}^{-}}\left(x_{1}^{*}\right)+\mathbf{L}_{2} \mathbf{f}_{20}^{+}\left(x_{1}^{*}\right)+\overline{\mathbf{L}_{2}} \overline{\mathbf{f}_{20}}\left(x_{1}^{*}\right)=0
$$

With the definition in (41), (47) reveals the first relation between $\Theta_{0}^{+}\left(x_{1}^{*}\right)$ and $\Theta_{0}^{-}\left(x_{1}^{*}\right)$, which is that

$$
\Theta_{0}^{+}\left(x_{1}^{*}\right)-\Theta_{0}^{-}\left(x_{1}^{*}\right)=0
$$

Using (43)-(46), (49) and the definition in (42), (48) reduce to the second relation between $\Phi_{0}^{+}\left(x_{1}^{*}\right)$ and $\Phi_{0}^{-}\left(x_{1}^{*}\right)$ as

$$
\Phi_{0}^{+}\left(x_{1}^{*}\right)+\overline{\mathbf{H}}^{-1} \mathbf{H} \Phi_{0}^{-}\left(x_{1}^{*}\right)=\overline{\mathbf{H}}^{-1}\left(\mathbf{B}_{2}+\overline{\mathbf{B}_{2}}\right) \Theta_{0}^{+}\left(x_{1}^{*}\right) .
$$

With the same procedures which were used to find (49) and (50), manipulations between (37) and (38) also give us two relations of $\Theta_{0}^{+}\left(x_{1}^{*}\right)$ and $\Theta_{0}^{-}\left(x_{1}^{*}\right)$ and of $\Phi_{0}^{+}\left(x_{1}^{*}\right)$ and $\Phi_{0}^{-}\left(x_{1}^{*}\right)$ as

$$
\begin{aligned}
\Theta_{1}^{+}\left(x_{1}^{*}\right)-\Theta_{1}^{-}\left(x_{1}^{*}\right)= & -Y_{+}\left(x_{1}^{*}\right)\left[\mathbf{L}_{1}\left\langle\left\langle\mu_{1}\right\rangle\right\rangle \mathbf{f}_{10}^{+}\left(x_{1}^{*}\right)+\overline{\mathbf{L}_{1}}\left\langle\left\langle\overline{\mu_{1}}\right\rangle \overline{\mathbf{f}_{10}^{-}}\left(x_{1}^{*}\right)\right]\right. \\
& +Y_{-}\left(x_{1}^{*}\right)\left[\mathbf{L}_{2}\left\langle\left\langle\mu_{2}\right\rangle\right\rangle \mathbf{f}_{20}^{\prime}\left(x_{1}^{*}\right)+\overline{\mathbf{L}_{2}}\left\langle\left\langle\overline{\mu_{2}}\right\rangle \overline{\mathbf{f}_{20}^{-}}\left(x_{1}^{*}\right)\right]\right.
\end{aligned}
$$

and 


$$
\begin{aligned}
\Phi_{1}^{+}\left(x_{1}^{*}\right)+\overline{\mathbf{H}}^{-1} \mathbf{H} \Phi_{1}^{-}\left(x_{1}^{*}\right)= & \overline{\mathbf{H}}^{-1}\left(\mathbf{B}_{2}+\overline{\mathbf{B}_{2}}\right) \Theta_{1}^{-}\left(x_{1}^{*}\right) \\
& -Y_{+}\left(x_{1}^{*}\right)\left[\mathbf{L}_{1}\left\langle\left\langle\mu_{1}\right\rangle\right\rangle \mathbf{f}_{10}^{+}\left(x_{1}^{*}\right)+\overline{\mathbf{L}_{1}}\left\langle\left\langle\overline{\mu_{1}}\right\rangle \overline{\mathbf{f}_{10}^{\prime}}\left(x_{1}^{*}\right)\right] .\right.
\end{aligned}
$$

Equations (49) and (50) will be used to find the solution to the order of $\varepsilon^{0},(51)$ and (52) were then used to find the solution to the order of $\varepsilon^{1}$. (49) and (50) are the homogeneous and general Hilbert's problems, respectively. Referring to the solutions of Hilbert's problems in England (1971), these problems can be solved without further difficulties. After obtaining the basic solution to the order of $\varepsilon^{0}$, the solution to the order of $\varepsilon^{1}$, however, can be solved from (51) and (52) when the profile of the perturbed-interface crack $Y_{ \pm}\left(x^{*}\right)$ is given. At first, the solution to the order of $\varepsilon^{0}$ must be solved because it is valid for different types of perturbed-interface cracks, hence, it is named basic solution.

Because $\Theta_{0}^{+}\left(x_{1}\right)=\Theta_{0}^{-}\left(x_{1}\right), \Theta_{0}^{+}\left(x_{1}^{*}\right)=\Theta_{0}^{-}\left(x_{1}^{*}\right)$ in eqn (49) and the remote stresses are constant, the potential vector function $\Theta_{0}(z)$ must be the form of

$$
\Theta_{0}(z)=l z,
$$

where $l$ is a complex constant vector.

From (43) and (44), we have

$$
\overline{\Phi_{k}}(z)=\overline{\mathbf{L}_{1}} \overline{\mathbf{f}_{1 k}}(z)=\overline{\mathbf{H}}^{-1} \mathbf{H} \Phi_{k}(z)-\overline{\mathbf{H}}^{-1}\left(\mathbf{B}_{2}+\overline{\mathbf{B}_{2}}\right) \Theta_{k}(z)
$$

Taking the product of (54) with the matrix $\overline{\mathbf{H}}$ from the lcft side, it becomes

$$
\overline{\mathbf{H}} \overline{\boldsymbol{\Phi}}_{k}(z)=\mathbf{H} \Phi_{k}(z)-\left(\mathbf{B}_{2}+\overline{\mathbf{B}}_{2}\right) \Theta_{k}(z)
$$

and its functional conjugate form is

$$
\mathbf{H} \Phi_{k}(z)=\mathbf{H} \overline{\Phi_{k}}(z)-\left(\overline{B_{2}}+\mathbf{B}_{2}\right) \overline{\Theta_{k}}(z) .
$$

Comparing (56) with (55), we have

$$
\overline{\Theta_{k}}(z)=-\Theta_{k}(z)
$$

Hence, from the knowledge of (53), $l$ is a pure imaginary vector.

According to the similar research for the Hilbert's problem in Suo (1990), the homogeneous solution to $(50)$ is

$$
\boldsymbol{\Phi}_{0}^{\mathbf{H}}(z)=\left(z^{2}-1\right)^{1 / 2}\left[a w\left(\frac{z-1}{z+1}\right)^{\mathbf{i} e}+b \bar{w}\left(\frac{z-1}{z+1}\right)^{-\mathbf{i e}}+c w_{3}\right]
$$

where $a, b$ and $c$ are complex constants, while the constant vectors $w$ and $w_{3}$ and the constant scalar $e$ are found to form three distinct eigenpairs

$$
(\mathbf{e}, \mathbf{w}), \quad(-\mathbf{e}, \overline{\mathbf{w}}), \quad\left(0, \mathbf{w}_{3}\right),
$$

satisfying

$$
\overline{\mathbf{H}} \mathbf{w}=\mathbf{e}^{2 \pi e} \mathbf{H w}, \quad \overline{\mathbf{H}}_{3}=\mathbf{H w}_{3} .
$$

Referring to the appendix in Ting (1986), the eigenroot $\mathbf{e}$ in (60) can be found. 


$$
\mathbf{e}=\frac{1}{2 \pi} \ln \frac{1+\zeta}{1-\zeta},
$$

where $\zeta=\left[-\frac{1}{2} \operatorname{tr}(\mathbf{M})^{2}\right]^{1 / 2}$ and $\mathbf{M}=\mathbf{i}(\mathbf{H}-\mathbf{H})(\mathbf{H}+\mathbf{H})^{-1}$.

The vectors $w$ and $w_{3}$ have the orthogonal properties in the sense

$$
\mathbf{w}^{\mathrm{T}} \mathbf{H} \mathbf{w}=\mathbf{w}^{\mathrm{T}} \mathbf{H} \mathbf{w}_{3}=\mathbf{w}^{\mathrm{T}} \mathbf{H} \mathbf{w}_{3}=\mathbf{0}
$$

and orthogonality conditions involving $\mathbf{A}$ can be obtained by taking the complex conjugation of the above.

With the obtained functions $\Theta_{0}(z)=l z$ in (53), we have $\Theta_{0}^{+}\left(x_{1}^{*}\right)=l x_{1}^{*}$ to substitute into $(50)$ for determining the particular solution $\Phi_{0}^{P}(z)$. After involving the particular solution, the complete solution to (50) would be

$$
\Phi_{0}(z)=\left(z^{2}-1\right)^{1 / 2}\left[a \mathbf{w}\left(\frac{z-1}{z+1}\right)^{i e}+\mathbf{b w}\left(\frac{z-1}{z+1}\right)^{-k e}+c \mathbf{w}_{3}\right]+(\mathbf{H}+\mathbf{H})^{-1}\left(\mathbf{B}_{2}+\overline{\mathbf{B}_{2}}\right) l z
$$

So far, the zeroth order potential functions $\Theta_{0}(z)$ and $\Phi_{0}(z)$ are both obtained, then with (43)-(46) we can also find $f_{10}(z)$ and $f_{20}(z)$ for deriving the physical quantities to the zeroth order from (7)-(10).

In order to determine the complex constants $a, b$ and $c$, we derive the expression of the remote stress vector $\left(\sigma_{2 j}^{\infty}\right)$, from (9).

$$
\begin{aligned}
\left(\sigma_{2 j}^{\infty}\right)_{1} & =\left.\left\{\sigma_{2 j}(z)\right\}_{1}\right|_{z \rightarrow \infty}=\left.\left[\mathbf{L}_{1} \mathbf{f}_{10}^{\prime}(z)+\overline{\mathbf{L}_{1}} \overline{\mathbf{f}_{10}^{\prime}(z)}\right]\right|_{z \rightarrow \infty}=\left.\left[\Phi_{0}^{\prime}(z)+\overline{\Phi_{0}^{\prime}(z)}\right]\right|_{z \rightarrow \infty} \\
& =\left(1+\lambda^{-1}\right)(a \mathbf{w}+\bar{a} \bar{w})+2 c \mathbf{w}_{3},
\end{aligned}
$$

where $a=\lambda \bar{b}$ and $c$ is real, due to that $\left(\sigma_{2 j}^{\infty}\right)_{1}$ is a real vector and $\lambda=\mathbf{e}^{2 e \pi}$.

The constants $a, b$ and $c$ then can be found by taking inner products of (64).

$$
a=\frac{\mathbf{w}^{\mathrm{T}} \mathbf{H}\left(\sigma_{2 j}^{\infty}\right)_{1}}{\left(1+\lambda^{-1}\right) \mathbf{w}^{\mathrm{T}} \mathbf{H} \mathbf{w}}, \quad b=\lambda^{-1} \bar{a} \quad \text { and } \quad c=\frac{\mathbf{w}_{3}^{\mathrm{T}} \mathbf{H}\left(\sigma_{2 j}^{\infty}\right)_{1}}{2 \mathbf{w}_{3}^{\mathrm{T}} \mathbf{H} \mathbf{w}_{3}} .
$$

For proving the identity $\left(\sigma_{2 j}^{\infty}\right)_{1}=\left(\sigma_{2 j}^{\infty}\right)_{2}=\sigma_{2 j}^{\infty}$, we can derive the expression of $\left(\sigma_{2 j}^{\infty}\right)_{2}$ from (9) and (45) with the same procedures used to find (64), then we can find the two expressions are identical. On the other hand, the expressions of $\left(\sigma_{1 j}^{\infty}\right)_{1}$ and $\left(\sigma_{1 j}^{\infty}\right)_{2}$ are also derived to be shown below in order to find the relationship among the remote stresses.

$$
\begin{aligned}
\left(\sigma_{1 j}^{\infty}\right)_{1}= & \left\{-\mathbf{L}_{1}\left\langle\left\langle\mu_{1}\right\rangle\right\rangle \mathbf{f}_{10}^{\prime}(z)-\left.\overline{\mathbf{L}_{1}}\left\langle\left\langle\overline{\mu_{1}}\right\rangle \overline{\mathbf{f}_{10}^{\prime}(z)}\right\}\right|_{z \rightarrow \infty}\right. \\
= & -\left\{\mathbf{L}_{1}\left\langle\left\langle\mu_{1}\right\rangle\right\rangle \mathbf{L}_{1}^{-1}\left(a \mathbf{w}+\lambda^{-1} \bar{a} \overline{\mathbf{w}}+c \mathbf{w}_{3}\right)+\overline{\mathbf{L}_{1}}\left\langle\left\langle\overline{\mu_{1}}\right\rangle\right\rangle \overline{\mathbf{L}_{1}^{-1}}\left(\bar{a} \bar{w}+\lambda^{-1} a \mathbf{w}+c \mathbf{w}_{3}\right)\right. \\
& \left.+\left[\mathbf{L}_{1}\left\langle\left\langle\mu_{1}\right\rangle\right\rangle \mathbf{L}_{1}^{-1}-\overline{\mathbf{L}_{1}}\left\langle\left\langle\overline{\mu_{1}}\right\rangle\right\rangle \overline{\mathbf{L}_{1}^{-1}}\right](\mathbf{H}+\mathbf{H})^{-1}\left(\mathbf{B}_{2}+\overline{\mathbf{B}_{2}}\right) l\right\}
\end{aligned}
$$

and

$$
\begin{aligned}
\left(\sigma_{1 j}^{\infty}\right)_{2}= & \left.\left\{-\mathbf{L}_{2}\left\langle\left\langle\mu_{2}\right\rangle\right\rangle \mathbf{f}_{20}^{\prime}(z)-\overline{\mathbf{L}_{2}}\left\langle\left\langle\overline{\mu_{2}}\right\rangle\right\rangle \overline{\mathbf{f}_{20}^{\prime}(z)}\right\}\right|_{z \rightarrow \infty} \\
= & -\left\{\mathbf{L}_{2}\left\langle\left\langle\mu_{2}\right\rangle\right\rangle \mathbf{L}_{2}^{-1}\left(\lambda^{-1} a \mathbf{w}+\overline{a w}+c \mathbf{w}_{3}\right)+\overline{\mathbf{L}_{2}}\left\langle\left\langle\overline{\mu_{2}}\right\rangle\right\rangle \overline{\mathbf{L}_{2}^{-1}}\left(\lambda^{-1} a w+a \mathbf{w}+c \mathbf{w}_{3}\right)\right. \\
& \left.+\left[\mathbf{L}_{2}\left\langle\left\langle\mu_{2}\right\rangle\right\rangle \mathbf{L}_{2}^{-1}-\overline{\mathbf{L}_{2}}\left\langle\left\langle\overline{\mu_{2}}\right\rangle\right\rangle \overline{\mathbf{L}_{2}^{-1}}\right](\mathbf{A}+\mathbf{H})^{-1}\left(\mathbf{B}_{1}+\overline{\mathbf{B}_{2}}\right) l\right\} .
\end{aligned}
$$

There are some identities in the Stroh formalism of anisotropic elasticity derived in Ting (1988). These identities are useful in obtaining a real form solution to two-dimensional 
anisotropic elasticity problems. One of the identities will be utilized to simplify (66) and (67) and is shown below:

$$
\mathbf{L}_{\alpha}\left\langle\left\langle\mu_{\alpha}\right\rangle\right\rangle \mathbf{L}_{\alpha}^{-1}=\mathbf{N}_{\alpha 1}^{\mathrm{T}}-\mathbf{i} \mathbf{N}_{\alpha 3} \mathbf{B}_{\alpha}
$$

and other identities are also derived here with the aid of (68) for the simplification purpose,

$$
\left[\mathbf{L}_{1}\left\langle\left\langle\mu_{1}\right\rangle\right\rangle \mathbf{L}_{\mathbf{1}}^{-1}-\overline{\mathbf{L}}_{1}^{-}\left\langle\left\langle\overline{\mu_{1}}\right\rangle\right\rangle \overline{\mathbf{L}_{1}^{-1}}\right](\overline{\mathbf{H}}+\mathbf{H})^{-1}\left(\mathbf{B}_{2}+\overline{\mathbf{B}_{2}}\right)=-\mathbf{i} 2 \mathbf{N}_{13}\left(\mathbf{L}_{1}+\mathbf{L}_{2}\right)^{-1}
$$

and

$$
\left[\mathbf{L}_{2}\left\langle\left\langle\mu_{2}\right\rangle\right\rangle \mathbf{L}_{2}^{-1}-\overline{\mathbf{L}_{2}}\left\langle\left\langle\overline{\mu_{2}}\right\rangle\right\rangle \overline{\mathbf{L}_{2}^{-1}}\right](\overline{\mathbf{H}}+\mathbf{H})^{-1}\left(\mathbf{B}_{1}+\overline{\mathbf{B}_{1}}\right)=-\mathbf{i} 2 \mathbf{N}_{23}\left(\mathbf{L}_{1}+\mathbf{L}_{2}\right)^{-1} .
$$

Here, in (68), (69) and (70), $\mathbf{L}_{\alpha_{x}}$ is one of the three real matrices $\mathbf{H}, \mathbf{I}, \mathbf{S}$, first introduced by Barnett and Lothe (1973), which is symmetric and positive definite. While $\mathbf{N}_{\alpha 1}$ and $\mathbf{N}_{\alpha 3}$, derived in Ting (1988), are presented as follows.

$$
\mathbf{N}_{x 1}=-\left[\begin{array}{lll}
r_{6} & 1 & s_{6} \\
r_{2} & 0 & s_{2} \\
r_{4} & 0 & s_{4}
\end{array}\right] \text { and } \quad \mathbf{N}_{x 3}=\left(\frac{1}{\Delta}\left[\begin{array}{ccc}
-\hat{S}_{55} & 0 & \hat{S}_{15} \\
0 & 0 & 0 \\
\hat{S}_{15} & 0 & -\hat{S}_{11}
\end{array}\right]\right),
$$

where

$$
\begin{aligned}
& \Delta=\hat{S}_{1 \mid} \hat{S}_{55}-\left(\hat{S}_{15}\right)^{2}>0, \\
& r_{m}=\left(-\hat{S}_{55} \hat{S}_{1 m}+\hat{S}_{15} \hat{S}_{5 m}\right) / \Delta \quad(m=6,2,4),
\end{aligned}
$$

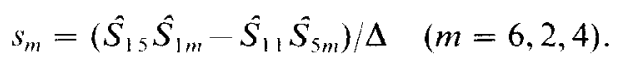

In the above, the reduced elastic compliance

$$
\hat{S}_{p q}=s_{p q}-s_{p 3} s_{3 q} / s_{33}
$$

is derived by letting

$$
\varepsilon_{3}=0=\sum_{q=1}^{6} s_{3 q} \sigma_{q}
$$

from the Hooke's law for the two-dimensional deformations. And solving for $\sigma_{3}$ from (73), we have

$$
\varepsilon_{p}=\sum_{q \neq 3}^{6} \hat{S}_{p q} \sigma_{u}, \quad p \neq 3
$$

Since

$$
\hat{S}_{p 3}=\hat{S}_{3 q}=0 \quad \text { for any } p,
$$

there is, in fact, no need to exclude $q=3$ in the summation of (74). Likewise, there is no need to exclude $p=3$ in (74) because it reduces to a trivial identity when $p=3$. The matrix $\hat{\mathrm{S}}$ considered as a $6 \times 6$ matrix has zero elements on the $3 \mathrm{rd}$ column and the $3 \mathrm{rd}$ row. If we remove the 3 rd column and the 3 rd row, the reduced $5 \times 5$ matrix $\hat{\mathbf{S}}$ can also connect the $5 \times 1$ matrices $\hat{\sigma}$ and $\hat{\varepsilon}$ which are obtained, respectively, by deleting from $\sigma$ and $\varepsilon$ the third element. (74) is equivalent to 


$$
\hat{\boldsymbol{\varepsilon}}=\hat{\mathbf{S}} \hat{\boldsymbol{\sigma}} \text {. }
$$

Hence, with (68), (69) and (70), the expressions (66) and (67) can be simplified to be

$$
\left(\sigma_{1 j}^{\infty}\right)_{1}=-\left\{\mathbf{N}_{11}^{\mathrm{T}} \sigma_{2 j}^{\infty}+2 \mathbf{N}_{13} \operatorname{Im}\left[\mathbf{B}_{1}\left(a \mathbf{w}+\lambda^{-1} \bar{a} \mathbf{w}+c \mathbf{w}_{3}\right)\right]-\mathbf{i} 2 \mathbf{N}_{13}\left(\mathbf{L}_{1}+\mathbf{L}_{2}\right)^{-1} l\right\}
$$

and

$$
\left(\sigma_{1 j}^{\infty}\right)_{2}=-\left\{\mathbf{N}_{21}^{\mathrm{T}} \sigma_{2 j}^{\infty}+2 \mathbf{N}_{23} \operatorname{Im}\left[\mathbf{B}_{2}\left(\lambda^{-1} a \mathbf{w}+\bar{a} \overline{\mathbf{w}}+c \mathbf{w}_{3}\right)\right]-\mathbf{i} 2 \mathbf{N}_{23}\left(\mathbf{L}_{1}+\mathbf{L}_{2}\right)^{-1} l\right\}
$$

Because the second rows of the $3 \times 3$ matrices $\mathbf{N}_{x 1}^{\mathrm{T}}$ and $\mathbf{N}_{x 3}$ are $(-1,0,0)$ and $(0,0,0)$, respectively, the second components of the vector equations (77) and (78) are trivial identities. Therefore, we can simplify (77) and (78) again by reducing the $3 \times 3$ matrices $\mathbf{N}_{\alpha 1}$ and $\mathbf{N}_{\alpha 3}$ to a $3 \times 2$ matrix $\mathbf{n}_{\alpha 1}$ and a $2 \times 2$ matrix $\mathbf{n}_{\alpha 3}$, respectively.

$$
\mathbf{n}_{\alpha 1}=-\left[\begin{array}{ll}
r_{6} & s_{6} \\
r_{2} & s_{2} \\
r_{4} & s_{4}
\end{array}\right]_{\alpha} \text { and } \quad \mathbf{n}_{\alpha 3}=\left(\frac{1}{\Delta}\left[\begin{array}{cc}
-\hat{\mathbf{S}}_{55} & \hat{\mathbf{S}}_{15} \\
\hat{\mathbf{S}}_{15} & -\hat{\mathbf{S}}_{11}
\end{array}\right]\right)_{\alpha}
$$

and with the proved identity $\operatorname{Im}\left[\mathbf{B}_{1}\left(a \mathbf{w}+\lambda^{-1} \bar{a} \overline{\mathbf{w}}+c \mathbf{w}_{3}\right)\right]=\operatorname{Im}\left[\mathbf{B}_{2}\left(\lambda^{-1} a \mathbf{w}+\bar{a} \overline{\mathbf{w}}+c \mathbf{w}_{3}\right)\right]$, which can be carried out easily with (60), eqns (77) and (78) are then expressed as

$$
\left[\begin{array}{l}
\sigma_{11}^{\infty} \\
\sigma_{13}^{\infty}
\end{array}\right]_{1}=-\left\{\mathbf{n}_{11}^{\mathrm{T}}\left[\begin{array}{l}
\sigma_{21}^{\infty} \\
\sigma_{22}^{\infty} \\
\sigma_{23}^{\infty}
\end{array}\right]+2 \mathbf{n}_{13}\left[\begin{array}{l}
\xi_{1} \\
\xi_{3}
\end{array}\right]-\mathbf{i} 2 \mathbf{n}_{13}\left[\begin{array}{l}
\vartheta_{1} \\
\vartheta_{3}
\end{array}\right]\right\}
$$

and

$$
\left[\begin{array}{l}
\sigma_{11}^{\infty} \\
\sigma_{13}^{\infty}
\end{array}\right]_{2}=-\left\{\mathbf{n}_{21}^{\mathrm{T}}\left[\begin{array}{l}
\sigma_{21}^{\infty} \\
\sigma_{22}^{\infty} \\
\sigma_{23}^{\infty}
\end{array}\right]+2 \mathbf{n}_{23}\left[\begin{array}{l}
\xi_{1} \\
\xi_{3}
\end{array}\right]-\mathbf{i} 2 \mathbf{n}_{23}\left[\begin{array}{l}
\vartheta_{1} \\
\vartheta_{3}
\end{array}\right]\right\} .
$$

Here, $\xi_{1}$ and $\xi_{3}$ are the first and the third components of the vector $\operatorname{Im}\left[\mathbf{B}_{1}\left(a \mathbf{w}+\lambda^{-1} \bar{a} \bar{w}+c \mathbf{w}_{3}\right)\right.$, as well as $\vartheta_{1}$ and $\vartheta_{3}$ are the first and the third components of the vector $\left(\mathbf{L}_{1}+\mathbf{L}_{2}\right)^{-1} l$, respectively. Then, letting $n_{13}^{-1}(80)$ be subtracted by $n_{23}^{-1}(81)$, we have the relation equation among the far-field stresses as

$$
\mathbf{n}_{13}^{-1}\left[\begin{array}{l}
\sigma_{11}^{\infty} \\
\sigma_{13}^{\infty}
\end{array}\right]_{1}-\mathbf{n}_{23}^{-1}\left[\begin{array}{l}
\sigma_{11}^{\infty} \\
\sigma_{13}^{\infty}
\end{array}\right]_{2}=\left(\mathbf{n}_{23}^{-1} \mathbf{n}_{21}^{\mathrm{T}}-\mathbf{n}_{13}^{-1} \mathbf{n}_{11}^{\mathrm{T}}\right)\left[\begin{array}{l}
\sigma_{21}^{\infty} \\
\sigma_{22}^{\infty} \\
\sigma_{23}^{\infty}
\end{array}\right],
$$

where

$$
\mathbf{n}_{\alpha 1}^{\mathrm{T}}=-\left[\begin{array}{lll}
r_{6} & r_{2} & r_{4} \\
s_{6} & s_{2} & s_{4}
\end{array}\right]_{\alpha} \text { and } \mathbf{n}_{\alpha 3}^{-1}=-\left[\begin{array}{ll}
\hat{\mathbf{S}}_{11} & \hat{\mathbf{S}}_{15} \\
\hat{\mathbf{S}}_{15} & \hat{\mathbf{S}}_{55}
\end{array}\right]_{\alpha}
$$

The relation equation (82) can be verified by the same result presented in Bassani and $\mathrm{Qu}$ (1990) and Qu and Bassani (1993). It has a concise form with the reduced elastic compliances $\hat{\mathbf{S}}$ as 


$$
\begin{aligned}
& {\left[\begin{array}{ll}
\hat{\mathbf{S}}_{11} & \hat{\mathbf{S}}_{15} \\
\hat{\mathbf{S}}_{15} & \hat{\mathbf{S}}_{55}
\end{array}\right]_{1}\left[\begin{array}{l}
\sigma_{11}^{\infty} \\
\sigma_{13}^{\infty}
\end{array}\right]_{1}-\left[\begin{array}{ll}
\hat{\mathbf{S}}_{11} & \hat{\mathbf{S}}_{15} \\
\hat{\mathbf{S}}_{15} & \hat{\mathbf{S}}_{55}
\end{array}\right]\left[\begin{array}{l}
\sigma_{11}^{\infty} \\
\sigma_{13}^{\infty}
\end{array}\right]_{2}} \\
& =\left(\left[\begin{array}{l}
\hat{\mathbf{S}}_{16} \\
\hat{\mathbf{S}}_{56}
\end{array}\right]_{2}-\left[\begin{array}{l}
\hat{\mathbf{S}}_{16} \\
\hat{\mathbf{S}}_{56}
\end{array}\right]\right) \sigma_{21}^{\infty}+\left(\left[\begin{array}{l}
\hat{\mathbf{S}}_{12} \\
\hat{\mathbf{S}}_{52}
\end{array}\right]_{2}-\left[\begin{array}{l}
\hat{\mathbf{S}}_{12} \\
\hat{\mathbf{S}}_{52}
\end{array}\right]\right) \sigma_{12}^{\infty}+\left(\left[\begin{array}{l}
\hat{\mathbf{S}}_{14} \\
\hat{\mathbf{S}}_{54}
\end{array}\right]_{2}-\left[\begin{array}{l}
\hat{\mathbf{S}}_{14} \\
\hat{\mathbf{S}}_{54}
\end{array}\right]\right) \sigma_{13}^{\infty} .
\end{aligned}
$$

When the materials are both isotropic, then (83) will reduce to

$$
\left(\sigma_{11}^{\infty}\right)_{1}=\frac{E_{1}\left(1-v_{2}^{2}\right)}{E_{2}\left(1-v_{1}^{2}\right)}\left(\sigma_{11}^{\infty}\right)_{2}+\left[\frac{v_{1}}{1-v_{1}}-\frac{E_{1}}{E_{2}} \frac{v_{2}\left(1+v_{2}\right)}{1-v_{1}^{2}}\right] \sigma_{22}^{\omega},
$$

which agrees with Rice and Sih (1965).

\section{SOLUTION TO THE ORDER OF $\varepsilon^{1}$}

Equations (51) and (52) can be used to find the solution to the order of $\varepsilon^{1}$ for any interesting perturbed-interface crack if the profiles of crack $Y_{ \pm}\left(x_{1}^{*}\right)$ is known. In this section, the regular case named holes with cusps $\left(Y_{+}-Y_{-} \geqslant 0\right)$ is examined. In this case the profile of the perturbed-interface crack will be simulated with

$$
Y_{+}\left(x_{1}^{*}\right)=\left(1-x_{1}^{* 2}\right)^{3 / 2} \quad \text { and } \quad Y_{-}\left(x_{1}^{*}\right)=\delta\left(1-x_{1}^{* 2}\right)^{3 / 2}
$$

where $-1 \leqslant \delta \leqslant 1$.

The functions selected in (85) have some characteristics. The power $3 / 2$ ensures that the crack faces have vanishing derivatives at \pm 1 , and makes such cracks regular. The parameter $\delta$ involved in the expression of $Y_{-}$can create some different configurations of regularly perturbed-interface cracks with its changeable values. Moreover, the expressions of the crack faces, which are used here to simulate the regularly perturbed-interface cracks for the purpose of demonstration, are essentially simple and useful to make the desired solutions easy to be carried out, without loss of generality.

As the study by Chen and Hsu (1995), the effect induced by the lateral far-field stresses will only exist in the first-order solution for the perturbed-interface crack. When the lateral far-field stresses are much more crucial than $\sigma_{2 j}^{\infty}$, then the first-order solution becomes an important consequence in the stress intensity factors. On the other hand, the effect induced by $\sigma_{2 j}^{\infty}$ in the first-order solution is therefore ignorable, because the zeroth-order solution which is resulted only by $\sigma_{2 j}^{\infty}$ can take over the whole concern for $\sigma_{2 j}^{\infty}$. Hence, in the following study only the lateral far-field stresses are involved in the derivation of first-order solution. Absolutely, such a simplification also makes the deriving process and the final solution elegant and delicate.

With the selected functions in (85) and the obtained zeroth-order solutions (51) and (52) can be presented as

$$
\begin{gathered}
\boldsymbol{\Theta}_{1}^{+}\left(x_{1}^{*}\right)-\Theta_{1}^{-}\left(x_{1}^{*}\right)=\left(1-x_{1}^{* 2}\right)^{3 / 2}\left[\left(\sigma_{1 j}^{\infty}\right)_{1}-\delta\left(\sigma_{1 j}^{\infty}\right)_{2}\right], \\
\Phi_{1}^{+}\left(x_{1}^{*}\right)+\overline{\mathbf{H}}^{-1} \mathbf{H} \Phi_{1}^{-}\left(x_{1}^{*}\right)=\overline{\mathbf{H}}^{-1}\left(\mathbf{B}_{2}+\overline{\mathbf{B}_{2}}\right) \Theta_{1}^{-}\left(x_{1}^{*}\right)+\left(1-x_{1}^{* 2}\right)^{3 / 2}\left(\sigma_{1 j}^{\infty}\right)_{1},
\end{gathered}
$$

where only the lateral stresses $\left(\sigma_{1 j}^{\infty}\right)_{1}$ and $\left(\sigma_{1 j}^{\infty}\right)_{2}$ are involved.

The following function will appear in the solution to (86) :

$$
K(z)=\left(z^{2}-1\right)^{3 / 2},
$$

which satisfies the condition 


$$
K^{+}\left(x_{1}^{*}\right)-K^{-}\left(x_{1}^{*}\right)=-\mathbf{i} 2\left(1-x_{1}^{*}\right)^{3 / 2}
$$

With the above function $K(z)$, the solution to (86) could be found, and it is

$$
\Theta_{1}(z)=\frac{\mathbf{i}}{2}\left(z^{2}-1\right)^{3 / 2}\left[\left(\sigma_{1 j}^{\infty}\right)_{1}-\delta\left(\sigma_{1 j}^{\infty}\right)_{2}\right]+\theta_{3} z^{3}+\theta_{2} z^{2}+\theta_{1} z
$$

where the vector polynomial $\theta_{3} z^{3}+\theta_{2} z^{2}+\theta_{1} z$ is so introduced that $\Theta_{1}(z)$ tends to $O\left(1 / z^{2}\right)$ as $z$ tends to infinity. The coefficient vectors are then determined as

$$
\theta_{3}=-\frac{\mathbf{i}}{2}\left[\left(\sigma_{1 j}^{\infty}\right)_{1}-\delta\left(\sigma_{1 j}^{\infty}\right)_{2}\right], \quad \theta_{2}=0 \quad \text { and } \quad \theta_{1}=\mathbf{i} \frac{3}{4}\left[\left(\sigma_{1 j}^{\infty}\right)_{1}-\delta\left(\sigma_{1 j}^{\infty}\right)_{2}\right]
$$

Writing eqn (87) in its components, or equivalently, taking the inner product of (87) with $\mathbf{w}^{\mathrm{T}} \mathbf{H}, \mathbf{w}^{\mathrm{T}}$ and $\mathbf{w}_{3}^{\mathrm{T}} \mathrm{H}$, we obtain

$$
\begin{gathered}
\phi_{11}^{+}\left(x_{1}^{*}\right)+\lambda^{-1} \phi_{11}^{-}\left(x_{1}^{*}\right)=\left(g_{1}-\frac{1}{2} h_{1}\right)\left(1-x_{1}^{* 2}\right)^{3 / 2}-\frac{\mathbf{i}}{2} h_{1} x_{1}^{* 3}+\mathbf{i} \frac{3}{4} h_{1} x_{1}^{*}, \\
\phi_{12}^{+}\left(x_{1}^{*}\right)+\lambda \phi_{12}^{-}\left(x_{1}^{*}\right)=\left(g_{2}-\frac{1}{2} h_{2}\right)\left(1-x_{1}^{* 2}\right)^{3 / 2}-\frac{\mathbf{i}}{2} h_{2} x_{1}^{* 3}+\mathbf{i} \frac{3}{4} h_{2} x_{1}^{*}, \\
\phi_{13}^{+}\left(x_{1}^{*}\right)+\phi_{13}^{-}\left(x_{1}^{*}\right)=\left(g_{3}-\frac{1}{2} h_{3}\right)\left(1-x_{1}^{* 2}\right)^{3 / 2}-\frac{\mathbf{i}}{2} h_{3} x_{1}^{* 3}+\mathbf{i} \frac{3}{4} h_{3} x_{1}^{*},
\end{gathered}
$$

where

$$
\begin{gathered}
h_{1} \mathbf{w}+h_{2} \overline{\mathbf{w}}+h_{3} \mathbf{w}_{3}=\mathbf{A}^{-1}\left(\mathbf{B}_{2}+\overline{\mathbf{B}_{2}}\right)\left[\left(\sigma_{1 j}^{\infty}\right)_{1}-\delta\left(\sigma_{1 j}^{\infty}\right)_{2}\right], \\
g_{1} \mathbf{w}+g_{2} \overline{\mathbf{w}}+g_{3} \mathbf{w}_{3}=\left(\sigma_{1 j}^{\infty}\right)_{1} .
\end{gathered}
$$

The components $h_{t s}$ and $g_{i s}$ are derived by taking the inner products of eqns (95) and (96).

$$
\begin{gathered}
h_{1}=\frac{\overline{\mathbf{w}}^{\mathrm{T}}\left(\mathbf{B}_{2}+\overline{\mathbf{B}_{2}}\right)\left[\left(\sigma_{1 j}^{\infty}\right)_{1}-\delta\left(\sigma_{1 j}^{\infty}\right)_{2}\right]}{\lambda \bar{w}^{\mathrm{T}} \mathbf{H w}}, \quad h_{2}=\frac{\overline{\mathbf{w}}^{\mathrm{T}}\left(\mathbf{B}_{2}+\overline{\mathbf{B}_{2}}\right)\left[\left(\sigma_{1 j}^{\infty}\right)_{1}-\delta\left(\sigma_{1 j}^{\infty}\right)_{2}\right]}{\lambda^{-1} w^{\mathrm{T}} \mathbf{H} \bar{w}} \\
h_{3}=\frac{\mathbf{w}_{3}^{\mathrm{T}}\left(\mathbf{B}_{2}+\overline{\mathbf{B}_{2}}\right)\left[\left(\sigma_{1 j}^{\infty}\right)_{1}-\delta\left(\sigma_{1 j}^{\infty}\right)_{2}\right]}{\mathbf{w}_{3}^{\mathrm{T}} \mathbf{H w}_{3}}
\end{gathered}
$$

and

$$
g_{1}=\frac{w^{\mathrm{T}} \mathbf{H}\left(\sigma_{1 j}^{\infty}\right)_{1}}{w^{\mathrm{T}} \mathbf{H} w}, \quad g_{2}=\frac{\mathbf{w}^{\mathrm{T}} \mathbf{H}\left(\sigma_{1 j}^{\infty}\right)_{1}}{\mathbf{w}^{\mathrm{T}} \mathbf{H}}, \quad g_{3}=\frac{\mathbf{w}_{3}^{\mathrm{T}} \mathbf{H}\left(\sigma_{1 j}^{\infty}\right)_{1}}{\mathbf{w}_{3}^{\mathrm{T}} \mathbf{H} \mathbf{w}_{3}}
$$

Hence, (92)-(94) are then scalar equations; they can be solved easily as done in Chen and Hsu (1995). Indeed, they are constructed from the known solutions for isotropic bimaterials, the complete solution is

$$
\Phi_{1}(z)=\phi_{11}(z) w+\phi_{12}(z) w+\phi_{13}(z) w_{3},
$$

where 


$$
\begin{aligned}
\phi_{11}(z)= & \mathbf{i} \frac{\lambda}{\lambda-1}\left(z^{2}-1\right)^{3 / 2}\left[1-\frac{z+\mathbf{i} 2 \varepsilon+1}{z+1}\left(\frac{z-1}{z+1}\right)^{\mathbf{i}}\right]\left(g_{1}-\frac{1}{2} h_{1}\right) \\
& -\mathbf{i} \frac{\lambda}{\lambda+1}\left(\frac{1}{2} z^{3}-\frac{3}{4} z\right) h_{1}+\left(z^{2}-1\right)^{1 / 2}\left(\frac{z-1}{z+1}\right)^{\mathbf{i}}\left(n_{12} z^{2}+n_{11} z+n_{10}\right), \\
\phi_{12}(z)= & \mathbf{i} \frac{1}{1-\lambda}\left(z^{2}-1\right)^{3 / 2}\left[1-\frac{z-\mathbf{i} 2 \varepsilon+1}{z+1}\left(\frac{z-1}{z+1}\right)^{-\mathbf{i} k}\right]\left(g_{2}-\frac{1}{2} h_{2}\right) \\
& -\mathbf{i} \frac{1}{\lambda+1}\left(\frac{1}{2} z^{3}-\frac{3}{4} z\right) h_{2}+\left(z^{2}-1\right)^{1 / 2}\left(\frac{z-1}{z+1}\right)^{-\mathbf{i} z}\left(n_{22} z^{2}+n_{21} z+n_{20}\right)
\end{aligned}
$$

and

$$
\begin{aligned}
\phi_{13}(z)= & \frac{1}{2 \pi}\left(z^{2}-1\right)^{3 / 2} \ln \left(\frac{z-1}{z+1}\right)\left(g_{3}-\frac{1}{2} h_{3}\right) \\
& -\mathbf{i} \frac{1}{4}\left(z^{3}-\frac{3}{2} z\right) h_{3}+\left(z^{2}-1\right)^{1 / 2}\left(n_{32} z^{2}+n_{31} z+n_{30}\right) .
\end{aligned}
$$

The vector polynomials $n_{1} z^{2}+n_{11} z+n_{10}, n_{22} z^{2}+n_{21} z+n_{20}$ and $n_{32} z^{2}+n_{31} z+n_{30}$ are also introduced such that all the component solutions, (98) $(100)$, tend to $O\left(1 / z^{2}\right)$ as $z$ tends to infinity. The coefficients are then determined.

$$
\begin{aligned}
& n_{12}=\mathbf{i} \frac{\lambda}{2(\lambda+1)} h_{1}, \quad n_{11}=\mathbf{i} 2 \varepsilon n_{12}=-\frac{\lambda}{\lambda+1} \varepsilon h_{1}, \\
& n_{10}=\mathbf{i} \frac{\lambda}{\lambda-1} \varepsilon(\varepsilon-\mathbf{i})\left(2 g_{1}-h_{1}\right)-\mathbf{i} \frac{\lambda}{\lambda+1}\left(\frac{1}{2}+2 \varepsilon^{2}\right) h_{1}, \quad n_{22}=\mathbf{i} \frac{1}{2(\lambda+1)} h_{2}, \\
& n_{21}=-\mathbf{i} 2 \varepsilon n_{22}=\frac{1}{\lambda+1} \varepsilon h_{2}, \quad n_{20}=\mathbf{i} \frac{1}{1-\lambda} \varepsilon(\varepsilon+\mathbf{i})\left(2 g_{2}-h_{2}\right)-\mathbf{i} \frac{1}{\lambda+1}\left(\frac{1}{2}+2 \varepsilon^{2}\right) h_{2}, \\
& n_{32}=\frac{\mathbf{i}}{4} h_{3}, \quad n_{31}=\frac{1}{\pi}\left(g_{3}-\frac{1}{2} h_{3}\right) \text { and } n_{30}=\frac{1}{2} n_{32}-\mathbf{i} \frac{3}{8} h_{3}=-\mathbf{i} \frac{1}{4} h_{3}=-n_{32} .
\end{aligned}
$$

So far, the solution to the regularly perturbed-interface crack of anisotropic bimaterial is obtained to the first-order of the unevenness of crack face. Then, the complex potential vector functions $f_{11}(z)$ and $f_{21}(z)$ can be determined from (43)-(46) with the found $\Theta_{1}(z)$ and $\Phi_{1}(z)$ for those physical quantities in both materials.

\section{STRESS INTENSITY FACTORS}

The traction in the bonded interface a distance $r$ ahead of the crack tip is given as

$$
\mathbf{t}(r)=(2 \pi r)^{-1 / 2}\left[K r^{i e} w+\bar{K} r^{-i e} \bar{w}+K_{3} w_{3}\right] .
$$

It reads that the interface traction at each fixed point $r$ can be decomposed into two components: one is along $w_{3}$ and the other is in the plane spanned by $\operatorname{Re}[w]$ and $\operatorname{Im}[w]$. This equation may be taken as the defining equation for the complex $K$ and real $K_{3}$. As $r$ approaches the tip, the $w_{3}$ component has a square root singularity and the planar component is oscillatory, with $K_{3}$ and $K$ measuring their intensities, respectively. The results are clearly the analogue of the corresponding ones for isotropic bimaterials. After deriving 
the expression of $t(r)$ with the obtained solutions in the last two sections, the stress intensity factors can be easily extracted by comparison with eqn (101).

The stress intensity factors derived from the zeroth-order solution are

$$
K=\sqrt{\pi 2^{-i e}}(1+\mathbf{i} 2 e)\left(1+\lambda^{-1}\right) a
$$

and

$$
K_{3}=\sqrt{\pi} 2 c
$$

which are induced only by $\sigma_{2 j}^{\infty}$ and the stress intensity factors derived from the first-order solution are

$$
\begin{aligned}
K & =\varepsilon \sqrt{\pi} 2^{-\mathbf{i e}}(1+\mathbf{i} 2 \varepsilon)\left(n_{12}+n_{11}+n_{10}+\overline{n_{22}}+\overline{n_{21}}+\overline{n_{20}}\right) \\
& =\varepsilon \sqrt{\pi} \frac{2^{1-\mathbf{i} e}}{1-\lambda} e(1+\mathbf{i} e)(1+\mathbf{i} 2 e)\left[\lambda h_{1}-(\lambda+1) g_{1}\right]
\end{aligned}
$$

and

$$
\begin{aligned}
K_{3} & =\varepsilon \sqrt{\pi}\left(n_{32}+n_{31}+n_{30}+\overline{n_{32}}+\overline{n_{31}}+\overline{n_{30}}\right)=\varepsilon \sqrt{\pi}\left(n_{31}+\overline{n_{31}}\right) \\
& =\varepsilon \frac{1}{\sqrt{\pi}}\left(2 g_{3}-h_{3}\right),
\end{aligned}
$$

where $\sigma_{11}^{\infty}$ and $\sigma_{13}^{\infty}$ are the only considered far-field stresses.

The structure of the near-tip fields around an interface crack has been identified, with only one real and one complex factor $K_{3}$ and $K$. In principle, for a given boundary value problem, these factors should be determined by the external geometry and load, and can be used in a similar way as the conventional stress intensity factors in Irwin's fracture mechanics. The results obtained in (102) and (103) are the basic solutions to the problem of finite interface crack of anisotropic bimaterial, but they are induced only by the far-field stresses $\sigma_{2 j}^{\infty}$. Once the stresses $\sigma_{2 j}^{\infty}$ diminish and the other stresses $\sigma_{11}^{\infty}$ and $\sigma_{13}^{\infty}$ dominate the fracture behavior of the perturbed-interface crack, then the corresponding stress intensity factors become the results presented in (104) and (105). The results in (104) and (105) have been multiplied by $\varepsilon$ which represents the unevenness of the crack faces. As the simple example proposed in Chen and Hsu (1995), the critical fracture parameters, such as $K_{3}^{\text {c }}$ and $K^{\mathrm{c}}$, can be found experimentally with eqns (102) and (103), then these critical values are used to determine the stresses $\left(\sigma_{11}^{\infty}\right)_{\text {critical }}$ and $\left(\sigma_{13}^{\infty}\right)_{\text {critical }}$ from (104) and (105) when these lateral stresses dominate the fracture behavior in some practical cases. In general, in eqn (101), the two eigenvectors $w$ and $w_{3}$ should be normalized to be dimensionless to give conventional dimensions for stress intensity factors. The different choices of normalization affect the definition of stress intensity factors by a real factor to $K_{3}$ and a complex factor to $K$.

For some necessities, the energy release rate $G$ is more useful than the stress intensity factors. Hence, with the expressions in (102)-(105) and the relationship between the energy release rate and the stress intensity factors, one will have the corresponding energy release rate for different loading cases. The energy release rate is so defined as

$$
G=\bar{w}^{\mathrm{T}}(H+\bar{H}) w|K|^{2} /\left(4 \cosh ^{2} \pi \varepsilon\right)+\frac{1}{8} w_{3}^{\mathrm{T}}(H+\bar{H}) w_{3} K_{3}^{2},
$$




\section{ON SOLUTIONS FOR ORTHOTROPIC MATERIALS}

In this section, bimaterials are considered with the $x y$-plane as a mirror plane, in which the in-plane and antiplane deformations are decoupled. They can be treated separately here. At first the authors will consider the antiplane-field. Hence, in eqn $(11), l_{3}(\mu)$ is identical zero for a material with such a symmetry. The characteristic equation for antiplane deformation becomes

$$
l_{2}(\mu) \equiv s_{55} \mu^{2}-2 s_{45} \mu+s_{44}=0 .
$$

The expression $s_{44} s_{55}-\left(s_{45}\right)^{2}$, a principal minor of the compliance matrix, is positive. Hence there are two complex conjugate roots to (107). According to the convention the root with a positive imaginary part is chosen, i.e.

$$
\mu_{3}=\left(s_{45}+\mathbf{i}\left(s_{44} s_{55}-s_{45}^{2}\right)^{1 / 2}\right) / s_{55} .
$$

Only one holomorphic function $f\left(z_{3}\right)$ is needed to represent antiplane deformations, with $z_{3}=x+\mu_{3} y$. The representations $(7)-(10)$ reduce to

$$
\begin{aligned}
u_{3} & =2 \operatorname{Re}\left[A f\left(z_{3}\right)\right], \quad T_{3}=-2 \operatorname{Re}\left[L f\left(z_{3}\right)\right], \\
\sigma_{23} & =2 \operatorname{Re}\left[L f^{\prime}\left(z_{3}\right)\right], \quad \sigma_{13}=-2 \operatorname{Re}\left[L \mu_{3} f\left(z_{3}\right)\right] .
\end{aligned}
$$

Now the defined $3 \times 3$ matrices $\mathbf{L}, \mathbf{A}$ and $\mathbf{B}$ reduce to scalars. They are

$$
\mathbf{L}=-1, \quad \mathbf{A}=\mathbf{i B}, \quad \mathbf{B}=\left(s_{44} s_{55}-s_{45}^{2}\right)^{1 / 2} .
$$

With these reduced results, the stress intensity factor derived from the zeroth-order solution in $(103)$ will reduces to

$$
K_{3}=\sqrt{\pi} \sigma_{23}^{\infty},
$$

which is derived by obtaining $c=\frac{1}{2} \sigma_{23}^{\infty}$ from (65) and the stress intensity factor derived from the first-order solution in (105) then also reduces to

$$
K_{3}=\varepsilon \frac{2}{\sqrt{\pi}\left(B_{1}+B_{2}\right)}\left[B_{1}\left(\sigma_{13}^{\infty}\right)_{1}+\delta B_{2}\left(\sigma_{13}^{\infty}\right)_{2}\right]
$$

Besides, for a homogeneous material with $x y$-plane as a mirror plane, the characteristic equation for in-plane deformation, specialized from (11), is

$$
l_{4}(\mu) \equiv s_{11} \mu^{4}-2 s_{16} \mu^{3}+\left(2 s_{12}+s_{66}\right) \mu^{2}-2 s_{26} \mu+s_{22}=0 .
$$

The roots of eqn (113), which have been shown by Lekhnitskii (1963), can never be real and thus they occur in two conjugate pairs. Assuming they are distinct, we can choose two different roots, $\mu_{1}$ and $\mu_{2}$, with positive imaginary parts, to each of which a complex variable $z_{j}=x+\mu_{j}$ is associated. The field quantities can be expressed by two holomorphic functions $f_{1}\left(z_{1}\right)$ and $f_{2}\left(z_{2}\right)$, as obtained by discarding $f_{3}\left(z_{3}\right)$ in (7)-(10).

The matrices $\mathbf{A}, \mathbf{L}, \mathbf{B}$ and $\mathbf{H}$ are $2 \times 2$ now the elements for $\mathbf{A}$ and $\mathbf{L}$ can be specialized from (12) and (13) with $\eta_{1}=\eta_{2}=0$, while

$$
\mathbf{B} \equiv \mathbf{i} \mathbf{A} \mathbf{L}^{-1}=\left[\begin{array}{cc}
s_{11} \mathbf{I m}\left(\mu_{1}+\mu_{2}\right) & -\mathbf{i}\left(\mu_{1} \mu_{2} s_{11}-s_{12}\right) \\
\mathbf{i}\left(\overline{\mu_{1}} \overline{\mu_{2}} s_{11}-s_{12}\right) & -s_{22} \mathbf{I m}\left(\mu_{1}^{-1}+\mu_{2}^{-1}\right)
\end{array}\right] .
$$

To gain more insight, the principal axes of each material are taken to be in $x$ and $y$ 
axes, since other orientations may be treated by in-plane rotations and the associated tensor rules shown in Ting (1982). Given an orthotropic solid, since $s_{16}=s_{26}=0$, only four elastic constants, $s_{11}, s_{22}, s_{12}$ and $s_{66}$, enter the plane problem formulation. Using the defined parameters by Suo (1990)

$$
\gamma=s_{11} / s_{22}, \quad \rho=\frac{1}{2}\left(2 s_{12}+s_{66}\right)\left(s_{11} s_{22}\right)^{-1 / 2},
$$

where the two parameters measure the anisotropy in the sense that $\gamma=1$ when the material has transversely cubic symmetry and $\gamma=\rho=1$ when the material is transversely isotropic. The positive definiteness of the strain energy density requires that

$$
\gamma>0 \text { and }-1<\rho<\infty \text {. }
$$

The characteristic equation (113) is then

$$
\gamma \mu^{4}+2 \rho \gamma^{1 / 2} \mu^{2}+1=0
$$

The roots with positive imaginary parts are

$$
\begin{aligned}
\mu_{1} & =\mathbf{i} \gamma^{-1 / 4}(n+m), \quad \mu_{2}=\mathbf{i} \gamma^{-1 / 4}(n-m), \quad \text { for } 1<\rho<\infty, \\
\mu_{1} & =\gamma^{-1 / 4}(\mathbf{i} n+m), \quad \mu_{2}=\gamma^{-1 / 4}(\mathbf{i} n-m), \quad \text { for }-1<\rho<1, \\
\mu_{1} & =\mu_{2}=\mathbf{i} \gamma^{-1 / 4}, \\
n & =\left[\frac{1}{2}(1+\rho)\right]^{1 / 2}, \quad m=\left|\frac{1}{2}(1-\rho)\right|^{1 / 2} .
\end{aligned}
$$

The matrix B for an orthotropic material, reduced from (114), is

$$
\mathbf{B}=\left[\begin{array}{cc}
2 n \gamma^{1 / 4}\left(s_{11} s_{22}\right)^{1 / 2} & \mathbf{i}\left(\left(s_{11} s_{22}\right)^{1 / 2}+s_{12}\right) \\
-\mathbf{i}\left(\left(s_{11} s_{22}\right)^{1 / 2}+s_{12}\right) & 2 n \gamma^{-1 / 4}\left(s_{11} s_{22}\right)^{1 / 2}
\end{array}\right]
$$

It is interesting to note that B is still well-behaved even if $\rho=1(A$ and $L$ are singular for this case). The matrix $\mathbf{H}$ for two orthotropic materials with aligne principal axes is

$$
\mathbf{H}=\left[\begin{array}{cc}
\mathbf{H}_{11} & \mathbf{i} \beta\left(\mathbf{H}_{11} \mathbf{H}_{22}\right)^{1 / 2} \\
-\mathbf{i} \boldsymbol{\beta}\left(\mathbf{H}_{11} \mathbf{H}_{22}\right)^{1 / 2} & \mathbf{H}_{22}
\end{array}\right],
$$

where

$$
\begin{aligned}
\mathbf{H}_{11} & =\left[2 n \gamma^{1 / 4}\left(s_{11} s_{22}\right)^{1 / 2}\right]_{\alpha=1}+\left[2 n \gamma^{1 / 4}\left(s_{11} s_{22}\right)^{1 / 2}\right]_{\alpha=2}, \\
\mathbf{H}_{22} & =\left[2 n \gamma^{-1 / 4}\left(s_{11} s_{22}\right)^{1 / 2}\right]_{\alpha=1}+\left[2 n \gamma^{-1 / 4}\left(s_{11}^{2} s_{22}\right)^{1 / 2}\right]_{\alpha=2}, \\
\left(\mathbf{H}_{11} \mathbf{H}_{22}\right)^{1 / 2} \beta & =\left[\left(s_{11} s_{22}\right)^{1 / 2}+s_{12}\right]_{\alpha=1}-\left[\left(s_{11} s_{22}\right)^{1 / 2}+s_{12}\right]_{\alpha=2} .
\end{aligned}
$$

Here $\beta$ is the same as $\zeta$ in (61) when the materials are orthotropic. The non-oscillatory fields can be obtained if $H$ is real, or $\beta=0$.

The oscillatory index $e$ now becomes

$$
e=(2 \pi)^{-1} \ln [(1+\beta) /(1-\beta)] .
$$

The normalized eigenvector, its definition form given dimensionlessly by Suo (1990) to give conventional dimensions for stress intensity factors, is 


$$
w=\left[-\frac{1}{2} \mathbf{i}, \frac{1}{2}\left(\mathbf{H}_{11} / \mathbf{H}_{22}\right)^{1 / 2}\right]^{\mathrm{T}} .
$$

Hence the complex constant $a$ can be determined with (119) and (122).

$$
a=\frac{\lambda}{1+\lambda}\left[\mathbf{i} \sigma_{21}^{\infty}+\left(\mathbf{H}_{22} / \mathbf{H}_{11}\right)^{1 / 2} \sigma_{22}^{\varkappa 1}\right]
$$

and the complex stress intensity factor in (102) is then determined as

$$
K=\sqrt{\pi} 2^{-\mathrm{i} e}(1+\mathbf{i} 2 e)\left[\mathbf{i} \sigma_{21}^{\prime \prime}+\left(\mathbf{H}_{22} / \mathbf{H}_{11}\right)^{1 / 2} \sigma_{22}^{\mu \nu}\right]
$$

With the component expression of the matrix $\mathbf{B}$ in (118), we will have the component expression of $\mathbf{B}+\overline{\mathbf{B}}$ for determining $h_{1}$.

$$
\mathbf{B}+\overline{\mathbf{B}}=\left[\begin{array}{cc}
4 n \gamma^{1.4}\left(s_{11} s_{22}\right)^{12} & 0 \\
0 & 4 n \gamma^{-1 / 4}\left(s_{1} s_{22}\right)^{1 / 2}
\end{array}\right]
$$

Then $h_{1}$ can be determined.

$$
\begin{aligned}
h_{1}= & \frac{4}{\lambda(1-\beta)}\left[\mathbf{i} \mathbf{H}_{11}^{-1}\left(n \sqrt{s_{11} s_{22} \gamma^{i, 4}}\right)_{x=2}\left\{\left(\sigma_{11}^{\infty}\right)_{1}-\delta\left(\sigma_{11}^{\times}\right)_{2}\right]\right. \\
& \left.+\frac{1}{\sqrt{\mathbf{H}_{1} \mathbf{H}_{22}}}\left(n \sqrt{s_{11} s_{22} \gamma^{1 / 4}}\right)_{x=2}\left\{\left(\sigma_{12}^{\times}\right)_{1}-\delta\left(\sigma_{12}^{\times}\right)_{2}\right\}\right] .
\end{aligned}
$$

Another constant $g_{1}$ can also be determined,

$$
g_{1}=\mathbf{i}\left(\sigma_{11}^{x}\right)_{1}+\left(\mathbf{H}_{22} / \mathbf{H}_{11}\right)^{12}\left(\sigma_{12}^{x}\right)_{1} .
$$

With the obtained $h_{1}$ and $g_{1}$ the complex stress intensity factor in (104) can also be determined without further difficulty.

\section{CONCLUSION}

Due to the agreement with reality, regularly perturbed-interface crack discussed in the present paper is a more practical model to analyze the real interface crack, especially for the fracture behavior dominated by lateral stresses. As the similar study by Chen and Hsu (1995), the basic techniques to analyze the regularly perturbed-interface crack of anisotropic bimaterial are constructed successfully. The primary techniques are LES representation and perturbation analysis. The LES representation named by Suo (1990) always provides a formalism for solving anisotropic elastic problems and now is still valid for treating the perturbed-interface crack. While, the perturbation analysis is used to divide the formulation of the interesting problem into two solving groups to the zeroth-order and to the firstorder, the interface crack is slightly perturbed. Then the regular perturbation procedures provide solutions to both the orders, and the corresponding stress intensity factors are obtained. With the finished study in this paper, the concept of interface fracture mechanics now includes treating an interface crack of anisotropic bimaterial, which has regularly perturbed surfaces.

The stress intensity factor is of the most concern in this paper. There are two groups of stress intensity factors determined to the zeroth-order and to the first-order, respectively. The stress intensity factors to the zeroth-order are induced only by $\sigma_{2 j}^{\infty}$ and the ones to the first-order are induced by $\sigma_{2 j,}^{\infty},\left(\sigma_{1 j}^{\infty}\right)_{1}$ and $\left(\sigma_{1 j}^{\infty}\right)_{2}$. When the remote stresses $\sigma_{2 j}^{\infty},\left(\sigma_{1 j}^{\infty}\right)_{1}$ and $\left(\sigma_{1 j}^{\infty}\right)_{2}$ are of the same order. Then it is enough to use the zeroth-order solution to represent the near-tip behavior as those analyses in past papers where the interface crack is assumed 
to be a flat thin-cut. Because the unevenness parameter $\varepsilon$ is small, neglecting the first-order effect will not give any serious consequences. That is why the interface crack can always be assumed to be a flat thin-cut. But when the lateral stresses $\left(\sigma_{1 j}^{\infty}\right)_{1}$ and $\left(\sigma_{1 j}^{\infty}\right)_{2}$ are much more crucial than $\sigma_{2 j}^{\infty}$, the order of magnitude of the first-order effect may be the same as or higher than the one of the zeroth-order effect. For this sake, the first-order solution becomes more and more important, provided that the fracture behavior is dominated by the lateral stresses. However, in the first order solution, the lateral stresses and $\sigma_{2 j}^{\infty}$ both give contributions. If the lateral stresses are so much more crucial, then $\sigma_{2 j}^{\infty}$ can be ignored in the firstorder solution. Nonetheless, we still have the effect induced by $\sigma_{2 j}^{\infty}$ in the zeroth order solution. Hence, $\sigma_{2 j}^{\infty}$ was taken away from the derivation of the first-order solution as the authors did in this paper. On the other hand, it also makes the final solution elegant and delicate to drop the remote stresses $\sigma_{2 j}^{\infty}$ out of the derivation of the first-order solution.

Acknowledgements - This work was supported by the National Science Council, R.O.C., under grant NSC-810401-E-002-549

\section{REFERENCES}

Barnett, D. M. and Lothe, J. (1973) Synthesis of the sextic and the integral formalism for dislocations, Green's function and surface waves in anisotropic elastic solids. Phys. Norv. 7, 13-19.

Barnett, D. M. and Lothe, J. (1985) Free surface (Rayleigh) waves in anisotropic elastic half-spaces: the surface impedance method. Proceedings of the Royal Society (London) A402, 135-152.

Bassani, J. L. and Qu, J. (1989) Finite crack on bimaterial and bicrystal interface. Journal of the Mechanics and Physics of Solids 37, 434-454.

Bassani, J. L. and Qu, J. (1990) Interface discontinuities and average bimaterial properties. Acta/Scripta Metallurgica Proceedings Series 4, 401-406.

Chen, C. H. and Hsu, J. (1995) The stress intensity factors of singularly and regularly perturbed-interface cracks of bimaterials (submitted to Mechanics of Materials).

Cherepanov, G. P. (1979) Mechanics of brittle materials. McGraw-Hill, New York.

Clements, D. L. (1971) A crack between dissimilar anisotropic media. International Journal of Engineering Science 9, 257-265.

Eshelby, J. D., Read, W. T. and Shockley, W. (1953) Anisotropic elasticity with applications to dislocation theory. Acta Metallica 1, 251-259.

Gotoh, M. (1967) Some problems of bonded anisotropic plates with cracks along the bond. International Journal of Fracture Mechanics 3, 253-260.

Lekhnitskii, S. G. (1963) Theory of elasticity of an anisotropic body. San Francisco : Holden-Day.

Ni, L. and Nemat-Nasser, S. (1991) Interface cracks in anisotropic dissimilar materials: an analytic solution. Quarterly Applied Mathematics 50, 305-322.

Qu, J. and Bassani, J. L. (1993) Interfacial fracture mechanics for anisotropic bimaterials. ASME Journal of Applied Mechanics 60, 422-431.

$\mathrm{Qu}$, J. and $\mathrm{Li}, \mathrm{Q}$. (1991) Interfacial dislocation and its applications to interface crack in anisotropic bimaterials. Journul of Elasticity 26, 169-195.

Rice, J. R. and Sih, G. C. (1965) Plane problems of cracks in dissimilar media. Journal of Applied Mechanics 32, 418-423.

Stroh, A. N. (1958) Dislocations and cracks in anisotropic elasticity. Philosophical Magazine 7, 625646.

Suo, Z. (1990) Singularities, interfaces and cracks in dissimilar anisotropic media. Proceedings of the Royal Society (London) A427, 331-358.

Tewary, V. K., Wagoner, R. H. and Hirth, J. P. (1989) Elastic Green's function for a composite solid with a planar crack in the interface. Journal of Material Research 4, 124-136.

Ting, T. C. T. (1982) Effects of change of reference coordinates on the stress analyses of anisotropic elastic materials. International Journal of Solids and Structures 18, 139-152.

Ting, T. C. T. (1986) Explicit solution and invariance of the singularities at an interface crack in anisotropic composites. International Journal of Solids and Structures 22, 965-983.

Ting, T. C. T. (1988) Some identities and the structure of $\mathbf{N}_{i}$ in the Stroh formalism of anisotropic elasticity. Quarterly Applied Mathematics 46, 109-120.

Ting, T. C. T. (1990) Interface cracks in anisotropic bimaterials. Journal of the Mechanics and Physics of Solids 38, 505-512.

Willis, J. R. (1971) Fracture mechanics of interface cracks. Journal of the Mechanics and Physics of Solids 19, 353368.

Wu, C. H. (1994) Regularly and singularly perturbed cracks. Quarterly Applied Mathematics 52, 529-543.

Wu, K. C. (1990) Stress intensity factor and energy release rate for interface cracks between dissimilar anisotropic materials. ASME Journal of Applied Mechanics 57, 894-900. 